\title{
Collider signals of the Mirror Twin Higgs boson through the hypercharge portal
}

\author{
Zackaria Chacko, ${ }^{1, *}$ Can Kilic, ${ }^{2, \dagger}$ Saereh Najjari, ${ }^{3,4, \ddagger}$ and Christopher B. Verhaaren $\circledast^{5, \S}$ \\ ${ }^{1}$ Maryland Center for Fundamental Physics, Department of Physics, \\ University of Maryland, College Park, Maryland 20742-4111, USA \\ ${ }^{2}$ Theory Group, Department of Physics, University of Texas at Austin, Austin, Texas 78712, USA \\ ${ }^{3}$ Theoretische Natuurkunde \& IIHE/ELEM, Vrije Universiteit Brussel, \\ Pleinlaan 2, 1050 Brussels, Belgium \\ ${ }^{4}$ PRISMA Cluster of Excellence \& Mainz Institute for Theoretical Physics, \\ Johannes Gutenberg University, 55099 Mainz, Germany \\ ${ }^{5}$ Center for Quantum Mathematics and Physics (QMAP), Department of Physics, \\ University of California, Davis, California 95616-5270, USA
}

\section{(Received 14 May 2019; published 29 August 2019)}

\begin{abstract}
We consider the collider signals arising from kinetic mixing between the hypercharge gauge boson of the Standard Model and its twin counterpart in the Mirror Twin Higgs model, in the framework in which the twin photon is massive. Through the mixing, the Standard Model fermions acquire charges under the mirror photon and the mirror $Z$ boson. We determine the current experimental bounds on this scenario and show that the mixing can be large enough to discover both the twin photon and the twin $Z$ at the $\mathrm{LHC}$, or at a future $100 \mathrm{TeV}$ hadron collider, with dilepton resonances being a particularly conspicuous signal. We show that, in simple models, measuring the masses of both the mirror photon and mirror $Z$, along with the corresponding event rates in the dilepton channel, overdetermines the system and can be used to test these theories.
\end{abstract}

DOI: $10.1103 /$ PhysRevD.100.035037

\section{INTRODUCTION}

With the discovery of the Higgs boson at the Large Hadron Collider (LHC) [1,2], all the particles predicted by the Standard Model (SM) have now been observed, and the search for new physics is well underway. Among the many puzzles this program may shed light on is the large hierarchy between the mass of the Higgs and the Planck scale. If a symmetry protects the Higgs mass from short distance quantum effects, then we expect new particles with masses of order the weak scale that are related to the SM particles by the symmetry. The symmetry partners of the top quark, the top partners, are expected to be particularly light. Despite increasingly sophisticated efforts, however, such particles have yet to be discovered at the LHC.

\footnotetext{
*zchacko@physics.umd.edu

${ }^{\dagger}$ kilic@physics.utexas.edu

saereh.najjari@vub.be

§cbverhaaren@ucdavis.edu
}

Published by the American Physical Society under the terms of the Creative Commons Attribution 4.0 International license. Further distribution of this work must maintain attribution to the author(s) and the published article's title, journal citation, and DOI. Funded by SCOAP.
The paradigm of neutral naturalness [3-17] addresses the hierarchy problem by incorporating color neutral top partners. Since color neutral particles are much more difficult for the LHC to discover, the bounds on this class of models remain relatively weak. In particular, the Mirror Twin Higgs (MTH) framework [3] protects the Higgs mass by employing fermionic top partners that are singlets under all the SM gauge groups. ${ }^{1}$ This protection results from a global symmetry in the Higgs sector combined with a discrete $\mathbb{Z}_{2}$ symmetry that exchanges each SM field with a mirror ("twin") copy that is charged under its own gauge groups. The electroweak (EW) gauge symmetries of both the SM and twin sectors are contained in the approximate global symmetry, which in the simplest realization of the model is $S U(4) \times U(1)$. This global symmetry is spontaneously broken at a scale $f$ down to $S U(3) \times U(1)$. The longitudinal modes of the $W$ and $Z$ vector bosons and the physical Higgs boson are among the resulting pseudoNambu-Goldsone bosons (pNGBs). The mass of the Higgs is protected against large radiative corrections by a combination of the nonlinearly realized global symmetry and the discrete $\mathbb{Z}_{2}$ twin symmetry.

\footnotetext{
${ }^{1}$ Recently, SM-singlet scalar top partner models have also been constructed $[14,15]$.
} 
After electroweak symmetry breaking the Higgs and its twin counterpart mix. If the $\mathbb{Z}_{2}$ symmetry were exact, the scales of electroweak symmetry breaking in the SM sector and the twin sector would be identical, $v=v^{\prime}$, where $v=$ $246 \mathrm{GeV}$ is the Higgs vacuum expectation value (VEV) in the SM, and we employ primes to denote the twin sector. Then, as a consequence of the mixing, the couplings of the Higgs boson to SM states would be suppressed, and there would be suppressed, with equal couplings to both visible and twin states. This, however, conflicts with existing experimental results on the couplings of the Higgs. Introducing soft breaking of the discrete $\mathbb{Z}_{2}$ symmetry allows the Higgs VEV $v$ to be small compared to $v^{\prime}$; thus the couplings of the physical Higgs boson to visible sector particles are close to their SM values, while the couplings to twin particles are suppressed. In this framework the ratio $v / f \equiv v / \sqrt{v^{2}+v^{12}}$ determines many observables, such as the ratio of the masses of the SM particles and their twin partners, as well as deviations in the couplings of the Higgs boson away from their SM values. The soft- $\mathbb{Z}_{2}$ breaking also introduces a tuning in the model, which scales as $(v / f)^{2}$. Experimental bounds on the Higgs couplings constrain $f / v \gtrsim 3$, while requiring the model be less than $10 \%$ tuned indicates $f / v \lesssim 6$ [18]. This provides a definite window for the twin particle masses: between 3 and 6 times the mass of their SM counterparts.

The MTH framework has been explored and expanded on in recent years. It has been shown that the breaking of the discrete $\mathbb{Z}_{2}$ symmetry can be realized spontaneously [19-23]. Ultraviolet completions have been constructed based on supersymmetry [24-30] (see also [31]), compositeness of the Higgs [32-34], and most recently using a "turtle" construction [35]. Composite MTH models have been shown to be consistent with precision electroweak constraints [36] and flavor bounds [37], and their collider signals have been studied [38,39].

The cosmology of the MTH, in its original incarnation, is problematic. In the early universe, Higgs-mediated interactions keep the mirror sector in thermal equilibrium with the SM down to temperatures of order a few GeV [4]. Then the twin photon and twin neutrinos give an overly large contribution to the total energy density in radiation, leading to conflict with the bounds on dark radiation from the cosmic microwave background and big bang nucleosynthesis. This problem can be solved if the model is extended to incorporate an asymmetric reheating process that contributes to the energy density in the SM degrees of freedom but not to the mirror sector [40,41]. To solve the problem, this mechanism must operate at late times, after the two sectors have decoupled. This can be realized without requiring additional breaking of the discrete $Z_{2}$ symmetry that relates the two sectors $[42,43]$. An alternative approach is to introduce hard breaking of the $Z_{2}$ into the twin sector Yukawa couplings, thereby altering the spectrum of mirror states [44-47]. This affects the number of degrees of freedom in the two sectors at the time of decoupling, allowing the cosmological bounds to be satisfied. Once this problem has been resolved, questions such as the nature of dark matter $[44,48]$, the origin of the baryon asymmetry [49,50], the order of the electroweak phase transition [51], and the implications of the MTH framework for large scale structure [52] can be addressed.

The cosmological challenges can also be solved by making the twin sector vectorlike [53]. An even more radical approach is to simply remove from the theory the two lighter generations of mirror fermions and the twin photon, which do not play a role in solving the little hierarchy problem. This construction, known as the Fraternal Twin Higgs (FTH) [54], gives rise to distinctive signatures at the LHC involving displaced vertices $[55,56]$ and admits several promising dark matter candidates [57-61].

In the MTH framework the discrete $\mathbb{Z}_{2}$ symmetry and the resulting gauge invariance under $[\mathrm{SU}(2) \times \mathrm{U}(1)]^{2}$ largely sequester the SM fields from their twin counterparts. The Higgs boson itself is the only low-energy portal between the two sectors that is guaranteed by construction. However, if the UV completion is weakly coupled, the radial mode associated with the breaking of the symmetry can be light enough to probe at current and future colliders [62-66]. In the absence of additional new fields [67], there is only one other renormalizable operator consistent with the symmetries that can link the two sectors: a kinetic mixing of the SM hypercharge gauge boson $B_{\mu}$ with its twin counterpart $B_{\mu}^{\prime}$,

$$
\frac{\epsilon}{2} B_{\mu \nu}^{\prime} B^{\mu \nu}
$$

Here $B_{\mu \nu}$ is the usual Abelian field strength. This operator has the effect of giving the SM quarks and leptons charges proportional to $\epsilon$ under the twin photon and twin $Z$, which are denoted $A^{\prime}$ and $Z^{\prime}$, respectively.

Apart from some discussion in the context of dark matter [57,59], previous analyses have largely neglected this mixing since it is not radiatively generated in the lowenergy theory to at least three-loop order [3,34]. An $\epsilon$ generated at the four-loop level is small enough to remain consistent with the very strong bounds on the photon mixing with another massless vector [68,69]; for a twin electron of mass of order $\mathrm{MeV}$ these bounds require $\epsilon \lesssim 10^{-9}$. However, ultraviolet completions of the MTH based on compositeness generically introduce states charged under both SM and twin gauge groups, with the result that $\epsilon$ is expected to receive one-loop corrections of order $10^{-2}-10^{-3}$. Such large values of $\epsilon$ can only be accommodated if the twin photon $A^{\prime}$ is massive.

In this paper we consider the collider signals arising from kinetic mixing between the hypercharge gauge boson of the SM and its twin counterpart in the MTH model, in the framework in which the twin photon is massive. We focus on the framework in which the discrete $\mathbb{Z}_{2}$ symmetry is 
only softly broken. We determine the current experimental bounds on this scenario and explore the possibility of discovering both the twin photon and the twin $Z$ at the LHC, or at a future $100 \mathrm{TeV}$ hadron collider such as the Future Circular Collider in hadron mode (FCC-hh). Although the phenomenology of new vector bosons that mix with the $\mathrm{U}(1)_{\mathrm{Y}}$ of the $\mathrm{SM}$ has been thoroughly explored [70-78], the MTH framework introduces several novel features.

A massive twin photon requires contributions to the masses of the mirror gauge bosons beyond those from electroweak symmetry breaking. This can be accomplished either by simply introducing a mass term for the twin hypercharge gauge boson or by extending the Higgs sector. In our analysis, we consider both cases. For the first case, we simply include an explicit Proca mass term for the twin hypercharge boson,

$$
\frac{m_{B^{\prime}}^{2}}{2} B_{\mu}^{\prime} B^{\prime \mu}
$$

where $m_{B^{\prime}}$ is a free parameter in the Lagrangian. Since a Proca mass term can be obtained from the Stückelberg construction, which is unitary and renormalizable, after gauge fixing, this theory is ultraviolet complete. The Proca mass can also be thought of as arising from the VEV of a new Higgs field that carries charge under twin hypercharge but not under twin $\mathrm{SU}(2)_{\mathrm{L}}$ [23]. If this Higgs field is heavy, it will decouple from the low energy spectrum, but if it carries only a small charge under $\mathrm{U}(1)_{\mathrm{Y}^{\prime}}$, the twin photon, though no longer massless, will still be light.

Alternatively, rather than include an explicit mass term for $B_{\mu}^{\prime}$, the twin photon can acquire a mass from the VEV of an additional Higgs field in the twin sector. For concreteness, we consider a scenario in which the Higgs content of the theory is extended to include two Higgs doublets in each of the SM and twin sectors [5,19-21]. The VEVs of the two doublets in the visible sector must be aligned to leave the photon massless, but the twin sector doublets are not so constrained. In particular, the VEVs of the two Higgs doublets in the twin sector must be somewhat misaligned to ensure that the twin photon has a mass.

As a consequence of the coupling in Eq. (1.1), the massive $Z^{\prime}$ and $A^{\prime}$ mix with the hypercharge gauge boson. Therefore the SM quarks and leptons acquire charges under the twin photon and twin $Z$, which can therefore be searched for at colliders. At hadron colliders, production through light quarks followed by decay into dileptons is a particularly promising channel. We find that values of the mixing parameter $\epsilon$ as small as $10^{-2}$ can potentially be probed at the LHC and FCC-hh.

In the softly broken $\mathbb{Z}_{2}$ scenario, a few physical parameters determine all the couplings in the model that are relevant for LHC searches. In particular, in the models we consider, the collider signals depend on only three parameters beyond those in the SM. Consequently, if more than three independent measurements can be performed, the parameters of the model are overdetermined, allowing these theories to be tested. Measuring the masses of the $A^{\prime}$ and $Z^{\prime}$, along with both their dilepton resonance production rates, achieves this goal.

In the following section we outline the interactions of the neutral vector bosons in the MTH framework and study their production rates and branching fractions. This is done for two scenarios: a model in which the twin hypercharge gauge boson is given an explicit Proca mass (THPM) and a twin two Higgs doublet model (T2HDM). In Sec. III, we determine the existing bounds on these two models from direct searches and from precision measurements. Then, in Sec. IV, we explore the discovery reach of the high luminosity LHC (HL-LHC) and a future $100 \mathrm{TeV}$ hadron collider and consider the prospects for testing the THPM and the T2HDM as the underlying origin of these resonances. We conclude in Sec. V.

\section{NEUTRAL VECTORS IN THE MIRROR TWIN HIGGS}

In this section we study the effects of kinetic mixing on the masses and couplings of the neutral vector bosons in the MTH model, in the scenario in which the twin photon is massive. We then explore the implications of mixing for the production cross sections and decay widths of these particles at colliders. We focus on the two case studies of a Proca mass for the twin hypercharge gauge boson and a two Higgs doublet version of the MTH model. For each case we obtain the production cross sections and branching fractions of the electrically neutral twin gauge bosons $A^{\prime}$ and $Z^{\prime}$ at the $\mathrm{LHC}$ and at a future $100 \mathrm{TeV}$ collider.

\section{A. Proca mass for twin hypercharge}

In this first case, we begin by considering the $\mathrm{SU}(2)_{\mathrm{L}} \times$ $\mathrm{U}(1)_{\mathrm{Y}} \times \mathrm{SU}(2)_{\mathrm{L}^{\prime}} \times \mathrm{U}(1)_{\mathrm{Y}^{\prime}}$ gauge sector,

$$
\begin{aligned}
\mathcal{L} \supset & -\frac{1}{2} \operatorname{Tr}\left[W_{\mu \nu} W^{\mu \nu}\right]-\frac{1}{2} \operatorname{Tr}\left[W_{\mu \nu}^{\prime} W^{\prime \mu \nu}\right]-\frac{1}{4} B_{\mu \nu} B^{\mu \nu} \\
& -\frac{1}{4} B_{\mu \nu}^{\prime} B^{\prime \mu \nu}+\frac{\epsilon}{2} B_{\mu \nu}^{\prime} B^{\mu \nu}+\frac{m_{B^{\prime}}^{2}}{2} B_{\mu}^{\prime} B^{\prime \mu},
\end{aligned}
$$

where primed fields belong to the twin sector and $\epsilon$ is the kinetic mixing parameter. For any spin-1 field $X_{\mu}$, we employ the notation

$$
X_{\mu \nu}=\partial_{\mu} X_{\nu}-\partial_{\nu} X_{\mu}
$$

to denote the field strength, with the usual generalization to the non-Abelian case.

Rather than working directly with $B^{\prime}$ and $W^{\prime 3}$, it is convenient to go over to the analogue of the familiar electroweak basis of the photon and $Z$ in the twin sector. 
Writing $s_{W} \equiv \sin \theta_{W}$ and $c_{W} \equiv \cos \theta_{W}$, where $\theta_{W}$ is the weak mixing angle, we define

$\bar{Z}_{\mu}^{\prime} \equiv c_{W} W_{\mu}^{\prime 3}-s_{W} B_{\mu}^{\prime}, \quad \bar{A}_{\mu}^{\prime} \equiv s_{W} W_{\mu}^{\prime 3}+c_{W} B_{\mu}^{\prime}$.

We find that, as expected, the Proca mass induces mass mixing between the two vector bosons,

$\frac{1}{2}\left(\begin{array}{ll}\bar{A}_{\mu}^{\prime} & \bar{Z}_{\mu}^{\prime}\end{array}\right)\left(\begin{array}{cc}c_{W}^{2} m_{B^{\prime}}^{2} & -s_{W} c_{W} m_{B^{\prime}}^{2} \\ -s_{W} c_{W} m_{B^{\prime}}^{2} & m_{\bar{Z}^{\prime}}^{2}+s_{W}^{2} m_{B^{\prime}}^{2}\end{array}\right)\left(\begin{array}{c}\bar{A}_{\mu}^{\prime} \\ \bar{Z}_{\mu}^{\prime}\end{array}\right)$.

Here $m_{\bar{Z}^{\prime}}^{2} \equiv\left(v^{\prime} / v\right)^{2} m_{Z_{0}}^{2}$, where $m_{Z_{0}}$ is the mass of the $Z$ boson in the SM. This mass matrix for the neutral twin sector vector bosons can be diagonalized by a simple rotation of the fields, with the mixing angle given by

$$
\sin 2 \phi=\frac{m_{B^{\prime}}^{2} s_{2 W}}{\sqrt{\left(m_{B^{\prime}}^{2}-m_{\bar{Z}^{\prime}}^{2}\right)^{2}+4 s_{W^{\prime}}^{2} m_{B^{\prime}}^{2} m_{\bar{Z}^{\prime}}^{2}}} .
$$

We label the lighter of the two mass eigenstates as $A_{\mu}^{\prime}$ and the heavier one as $Z_{\mu}^{\prime}$. Note that in the limit $m_{B^{\prime}}^{2} \gg m_{\bar{Z}^{\prime}}^{2}$, Eq. (2.5) implies that $\phi=\theta_{W}$. Essentially, this undoes the weak mixing, meaning that for large $m_{B^{\prime}}$, the mass eigenstates are, to a good approximation, simply the $W_{3}^{\prime}$ and the $B^{\prime}$ with masses $m_{\bar{Z}^{\prime}} c_{W}$ and $m_{B^{\prime}}$, respectively. In this limit the $Z^{\prime}$, which has its mass set by $m_{B^{\prime}}$, couples more strongly to the visible sector than the lighter $A^{\prime}$, which decouples from the SM as $m_{B^{\prime}}$ increases. Additional details are given in Appendix A.

In the opposite limit, $m_{B^{\prime}}^{2} \ll m_{\bar{Z}^{\prime}}^{2}$, the mixing angle $\phi$ tends to zero. The mass eigenstates are then, to a good approximation, simply $\bar{A}_{\mu}^{\prime}$ and $\bar{Z}_{\mu}^{\prime}$, with masses $m_{B^{\prime}} c_{W}$ and $m_{\bar{Z}^{\prime}}$, respectively. In this limit it is the lighter eigenstate, the $A^{\prime}$, which couples more strongly to the visible sector, while the couplings of the heavier $Z^{\prime}$ are suppressed by a relative factor of $\tan \theta_{W}$.

To analyze the experimental signals, we must transform from Eq. (2.1) to a basis diagonal in both mass and kinetic terms. This is accomplished by first performing a shift of $B$ and then rotating into the mass basis. The details of this procedure are given in Appendix A, where we obtain the field transformation to leading order in $\epsilon$. The couplings of the diagonalized fields to SM and twin particles are provided in Appendix B, again to leading order in $\epsilon$. However, this perturbative analysis breaks down near mass degeneracies, where one of the twin sector gauge bosons becomes close in mass to the SM $Z$ boson. Therefore the results we present have been obtained by diagonalizing the system numerically. For small $\epsilon$, away from mass degeneracies, the mass eigenvalues are close to the values obtained by diagonalizing the matrix Eq. (2.4). The mass eigenvalues as a function of $m_{B^{\prime}}$, for the benchmark values $f / v=3$ and 5 and $\epsilon=0.1$, are shown in Fig. 1. We see that

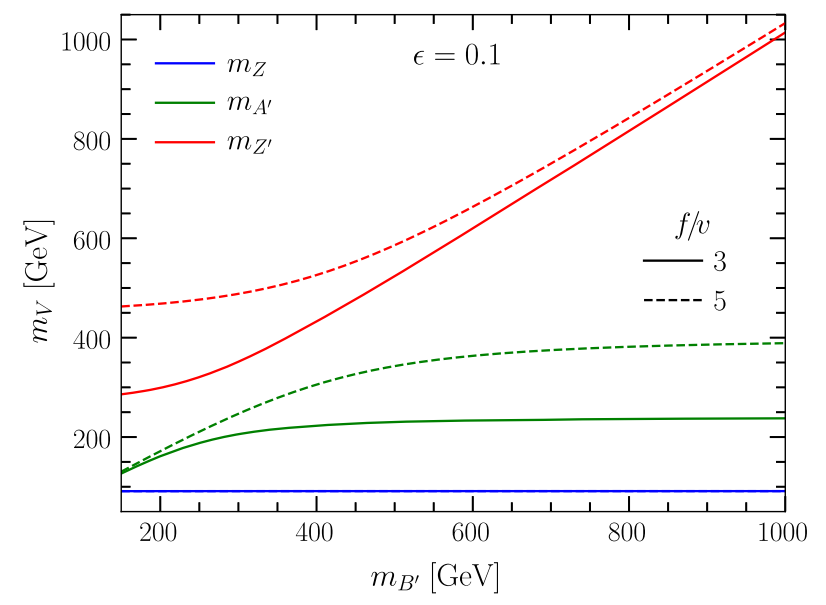

FIG. 1. The mass eigenvalues for the twin neutral vector bosons as a function of the Proca mass $m_{B^{\prime}}$ in the THPM model for $\epsilon=0.1$ and $f / v=3$ and 5 .

for large $m_{B^{\prime}}$, the mass of the heavier $Z^{\prime}$ is close to $m_{B^{\prime}}$, while that of the lighter $A^{\prime}$ asymptotes to $\left(v^{\prime} / v\right) m_{W}$.

After diagonalization the SM fermions acquire charges under the $A^{\prime}$ and the $Z^{\prime}$. This allows the twin vector bosons to be produced and detected at colliders. The production cross sections of the $A^{\prime}$ and $Z^{\prime}$ at the $13 \mathrm{TeV}$ LHC and at a $100 \mathrm{TeV}$ future hadron collider are shown in Fig. 2, for $\epsilon=0.1$ and $f / v=3$ and 5 . We see that for small values of its mass, the $A^{\prime}$ cross section is large but drops off quickly as the mass increases. This occurs because, for large $m_{B^{\prime}}$, the $A^{\prime}$ is almost entirely composed of $W_{3}^{\prime}$, which does not mix directly with SM hypercharge. Consequently, as $m_{B^{\prime}}$ increases, the cross section plummets. In contrast, the production cross section of the $Z^{\prime}$ remains sizable even for masses in the $\mathrm{TeV}$ range.

The corresponding branching fractions are shown in Fig. 3. As expected, decays to twin fermions dominate the width. Nevertheless, the branching fractions into SM fermions can be large enough for discovery in a clean resonant channel such as dileptons. Note that for large values of $m_{B^{\prime}}$, the branching fraction of $A^{\prime}$ into dileptons is much smaller than the corresponding $Z^{\prime}$ branching fraction. These hierarchies in the production cross sections and dilepton branching ratios translate into a much greater discovery potential for the $Z^{\prime}$ at colliders in most of the parameter space, as we show below.

\section{B. Twin two Higgs doublet model}

We now turn to the twin two Higgs doublet model. We label the two visible sector Higgs doublets as $H_{1}$ and $H_{2}$, and their twin counterparts as $H_{1}^{\prime}$ and $H_{2}^{\prime}$. Since the photon is massless, the VEVs of $H_{1}$ and $H_{2}$ must be aligned. However, the VEVs of the doublets in the twin sector need to be misaligned if they are to give mass to both the neutral gauge bosons in the twin sector. 

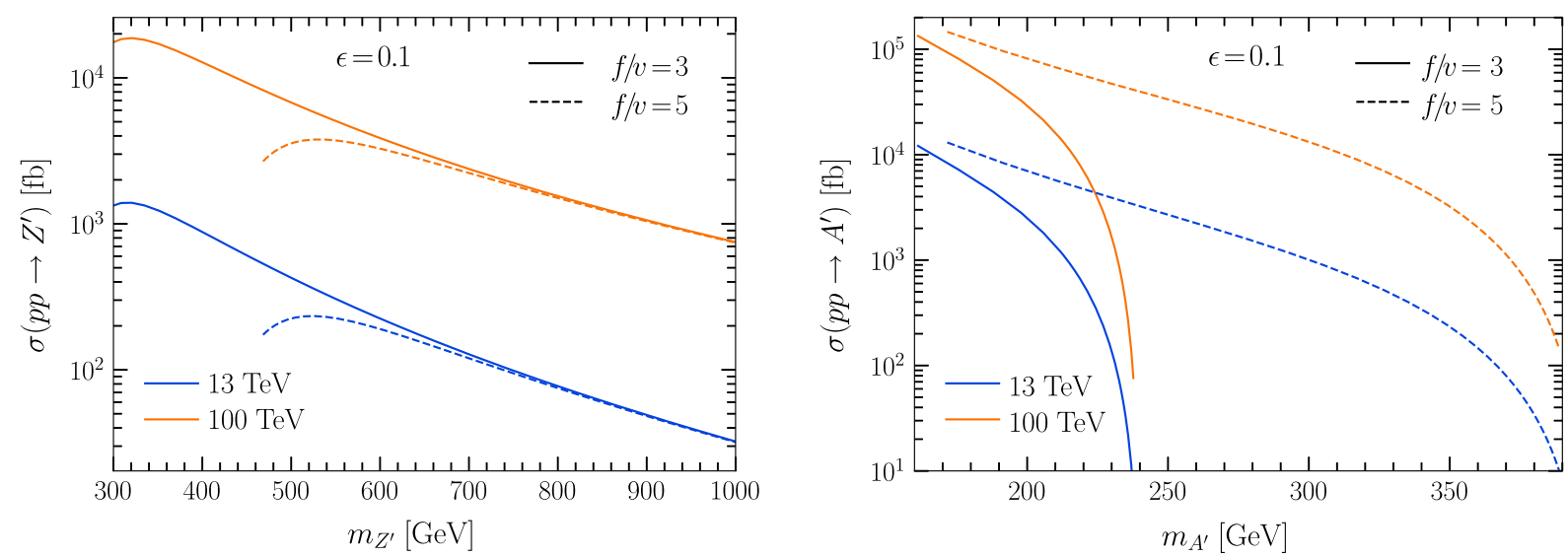

FIG. 2. Production cross section of the $Z^{\prime}$ (left) and $A^{\prime}$ (right) in the THPM model at the LHC and at a future $100 \mathrm{TeV}$ hadron collider for $\epsilon=0.1$ and $f / v=3$ and 5 .
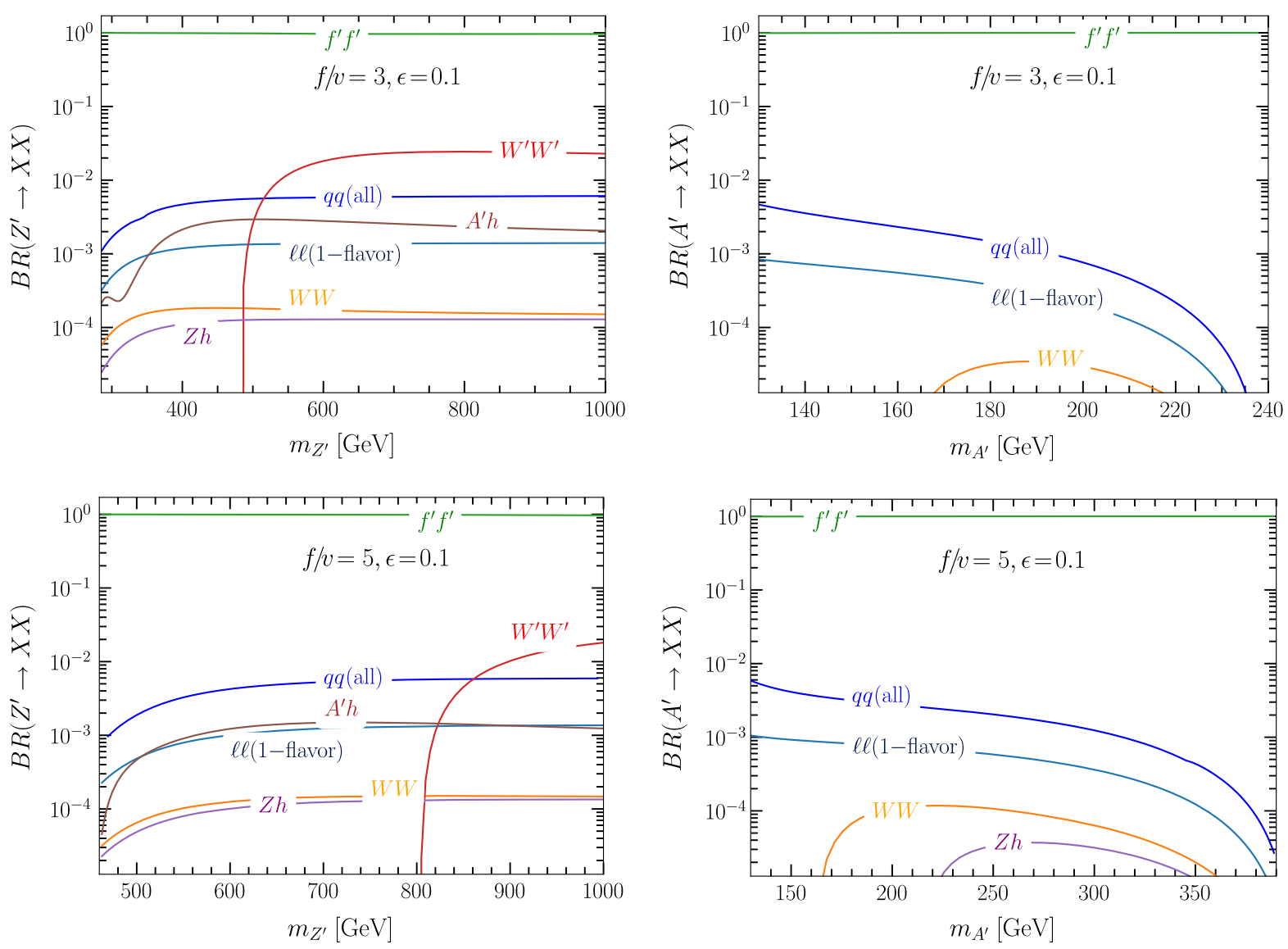

FIG. 3. Branching ratios of the $Z^{\prime}$ (left) and $A^{\prime}$ (right) for the THPM model for $\epsilon=0.1$ and $f / v=3(5)$ for the top (bottom) row. The light blue curve is for a single SM lepton flavor, while the dark blue curve corresponds to all six quark flavors.

The alignment of the two twin doublet VEVs can be quantified in many ways. For instance, the quantity

$$
\frac{\left|H_{1}^{\prime \dagger} H_{2}^{\prime}\right|^{2}}{\left(H_{1}^{\prime \dagger} H_{1}^{\prime}\right)\left(H_{2}^{\prime \dagger} H_{2}^{\prime}\right)}
$$

tends to one in the limit of perfect alignment and to zero in the limit of perfect antialignment. The limit of perfect alignment does not serve our purposes since it leaves one vector boson massless, but any other configuration will result in both the neutral gauge bosons in the twin sector acquiring masses. For concreteness, we restrict our 
attention to the limit of perfect antialignment in the twin sector. We further assume that of the two visible sector doublets, only $H_{1}$ acquires a VEV, while $\left\langle H_{2}\right\rangle=0$. It is straightforward to construct a potential for the scalar sector that gives rise to these features. While the potential for $\mathrm{H}_{2}$ and $H_{2}^{\prime}$ must respect the discrete $\mathbb{Z}_{2}$ twin symmetry, it need not obey the global $\mathrm{SU}(4) \times \mathrm{U}(1)$ symmetry. Therefore the scalar states in $\mathrm{H}_{2}$, the second visible sector doublet, are not required to be pNGBs and can therefore naturally be heavy.

The fact that both $H_{1}^{\prime}$ and $H_{2}^{\prime}$ acquire VEVs implies that there are two additional pNGB states in the mirror sector. If these states are to acquire a mass, there must be interactions that couple $H_{1}^{\prime}$ and $H_{2}^{\prime}$ in the potential for the scalar fields. We can lift these additional pNGBs from the low energy theory by including the term

$$
V=\lambda\left(\left|H_{1}^{\dagger} H_{2}\right|^{2}+\left|H_{1}^{\prime \dagger} H_{2}^{\prime}\right|^{2}\right)
$$

in the scalar potential. These terms respect the discrete $\mathbb{Z}_{2}$ symmetry and therefore do not generate a large mass for the light Higgs. For $\lambda \gtrsim 1$, these states acquire masses above those of the twin gauge bosons, and we can neglect their dynamics at low energies.

We can then write the VEVs of the Higgs fields as

$$
\begin{aligned}
& H_{1}=\left(\begin{array}{c}
0 \\
v / \sqrt{2}
\end{array}\right), \quad H_{1}^{\prime}=\left(\begin{array}{cc}
0 & \\
f_{1} \cos \vartheta & \sqrt{2}
\end{array}\right), \\
& H_{2}=\left(\begin{array}{l}
0 \\
0
\end{array}\right), \quad H_{2}^{\prime}=\left(\begin{array}{c}
f_{2} / \sqrt{2} \\
0
\end{array}\right) \text {, }
\end{aligned}
$$

where $\sin \vartheta=v / f_{1}$. The mass terms for the gauge bosons arise from the kinetic terms for the Higgs bosons,

$$
\mathcal{L} \supset\left|D_{\mu} H_{1}\right|^{2}+\left|D_{\mu} H_{2}\right|^{2}+\left|D_{\mu}^{\prime} H_{1}^{\prime}\right|^{2}+\left|D_{\mu}^{\prime} H_{2}^{\prime}\right|^{2} .
$$

From this we obtain the usual mass terms for the visible sector gauge bosons,

$$
\frac{g^{2} v^{2}}{4} W_{\mu}^{+} W^{-\mu}+\frac{g^{2} v^{2}}{8 c_{W}^{2}} Z_{\mu} Z^{\mu}
$$

The mass matrix for the twin sector is more complicated. In this case we find

$$
\begin{aligned}
& \frac{g^{2}}{4}\left(f_{1}^{2} \cos ^{2} \vartheta+f_{2}^{2}\right) W_{\mu}^{\prime+} W^{\prime-\mu}+\frac{g^{2}}{2} s_{W}^{2} f_{2}^{2} \bar{A}_{\mu}^{\prime} \bar{A}^{\prime \mu} \\
& \quad+\frac{g^{2} s_{W}}{2 c_{W}} f_{2}^{2} c_{2 W} \bar{A}_{\mu}^{\prime} \bar{Z}^{\prime \mu}+\frac{g^{2}}{8 c_{W}^{2}}\left[f_{1}^{2} \cos ^{2} \vartheta+f_{2}^{2} c_{2 W}^{2}\right] \bar{Z}_{\mu}^{\prime} \bar{Z}^{\prime \mu},
\end{aligned}
$$

where $\cos n \theta_{W} \equiv c_{n W}$. It is convenient to define $m_{\bar{Z}^{\prime}}=$ $m_{Z_{0}} \cot \vartheta$, where $m_{Z_{0}}$ is the $Z$ mass in the SM and
$m_{\bar{A}^{\prime}}=g f_{2} s_{W}$. Expressed in terms of these variables, the mass matrix is given by $\left(t_{2 W} \equiv \tan 2 \theta_{W}\right)$

$\frac{1}{2}\left(\begin{array}{ll}\bar{A}_{\mu}^{\prime} & \bar{Z}_{\mu}^{\prime}\end{array}\right)\left(\begin{array}{cc}m_{\bar{A}^{\prime}}^{2} & m_{\bar{A}^{\prime}}^{2} / t_{2 W} \\ m_{\bar{A}^{\prime}}^{2} / t_{2 W} & m_{\bar{Z}^{\prime}}^{2}+m_{\bar{A}^{\prime}}^{2} / t_{2 W}^{2}\end{array}\right)\left(\begin{array}{c}\bar{A}_{\mu}^{\prime} \\ \bar{Z}_{\mu}^{\prime}\end{array}\right)$,

which admits no massless state for $m_{\bar{A}^{\prime}}^{2}>0$.

From this point, the determination of the physical states and couplings in this model proceeds just as in the THPM model, with an example of how the physical masses depend on $m_{\bar{A}^{\prime}}$ given in Fig. 4. The mixing angle that diagonalizes the mass matrix in Eq. (2.12) is now given by

$$
\sin 2 \phi=-\frac{m_{\bar{A}^{\prime}}^{2} s_{4 W}}{\sqrt{\left(m_{\bar{Z}^{\prime}}^{2} s_{2 W}^{2}+c_{4 W} m_{\bar{A}^{\prime}}^{2}\right)^{2}+s_{4 W}^{2} m_{\bar{A}^{\prime}}^{4}}} .
$$

In the limit $m_{\bar{A}^{\prime}}^{2} \ll m_{\bar{Z}^{\prime}}^{2}$, the mixing angle $\phi$ tends to $-\pi / 2$, and the mass eigenstates become, to a good approximation, just $\bar{A}_{\mu}^{\prime}$ and $\bar{Z}_{\mu}^{\prime}$, with masses $m_{\bar{A}^{\prime}}$ and $m_{\bar{Z}^{\prime}}$, respectively. In this limit the lighter eigenstate, the $A^{\prime}$, couples more strongly to the visible sector, while the couplings of the heavier $Z^{\prime}$ are suppressed by a relative factor of $\tan \theta_{W}$. In the opposite limit, $m_{\bar{A}^{\prime}}^{2} \gg m_{\bar{Z}^{\prime}}^{2}$, the mass eigenvalues are given by $m_{\bar{Z}^{\prime}} \sin 2 \theta_{W}$ and $m_{\bar{A}^{\prime}} / \sin 2 \theta_{W}$. In this limit it is again the lighter eigenstate, the $A^{\prime}$, that couples more strongly to the visible sector, while the couplings of the heavier $Z^{\prime}$ are suppressed by the same relative factor of $\tan \theta_{W}$. This is very different from the THPM model, in which the couplings of the $A^{\prime}$ to the visible sector vanish in the limit that the $Z^{\prime}$ is heavy.

At the intermediate value $m_{\bar{A}^{\prime}}=s_{2 W} m_{\bar{Z}^{\prime}}$, corresponding to $\phi=\theta_{W}-\pi / 2$, the $\bar{Z}^{\prime}$ field rotates into $W^{\prime 3}$, which means it is orthogonal to $B^{\prime}$. As we see in Figs. 5 and 6, this causes the $Z^{\prime}$ to completely decouple from the visible sector,

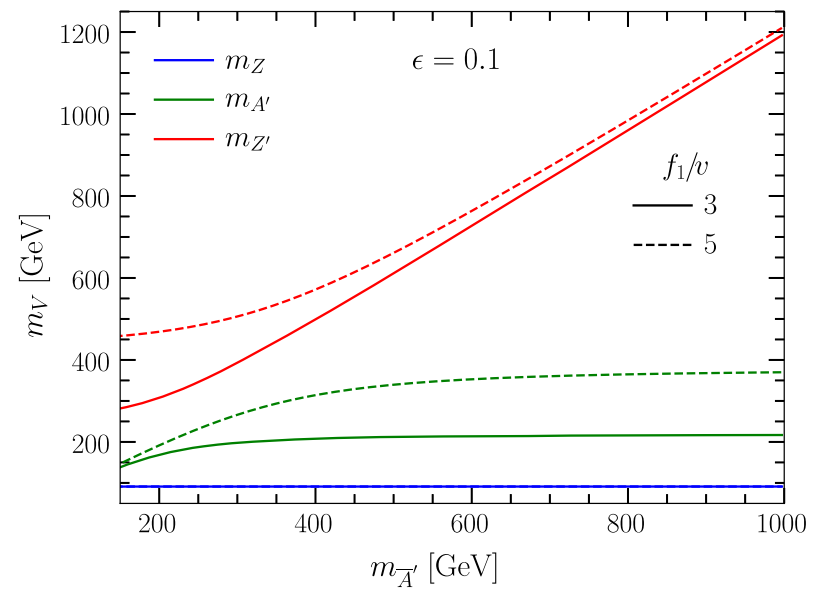

FIG. 4. The mass eigenvalues for the twin neutral vector bosons as a function of the twin photon mass $m_{\bar{A}^{\prime}}$ in the T2HDM for $\epsilon=0.1$ and $f_{1} / v=3$ and 5 . 

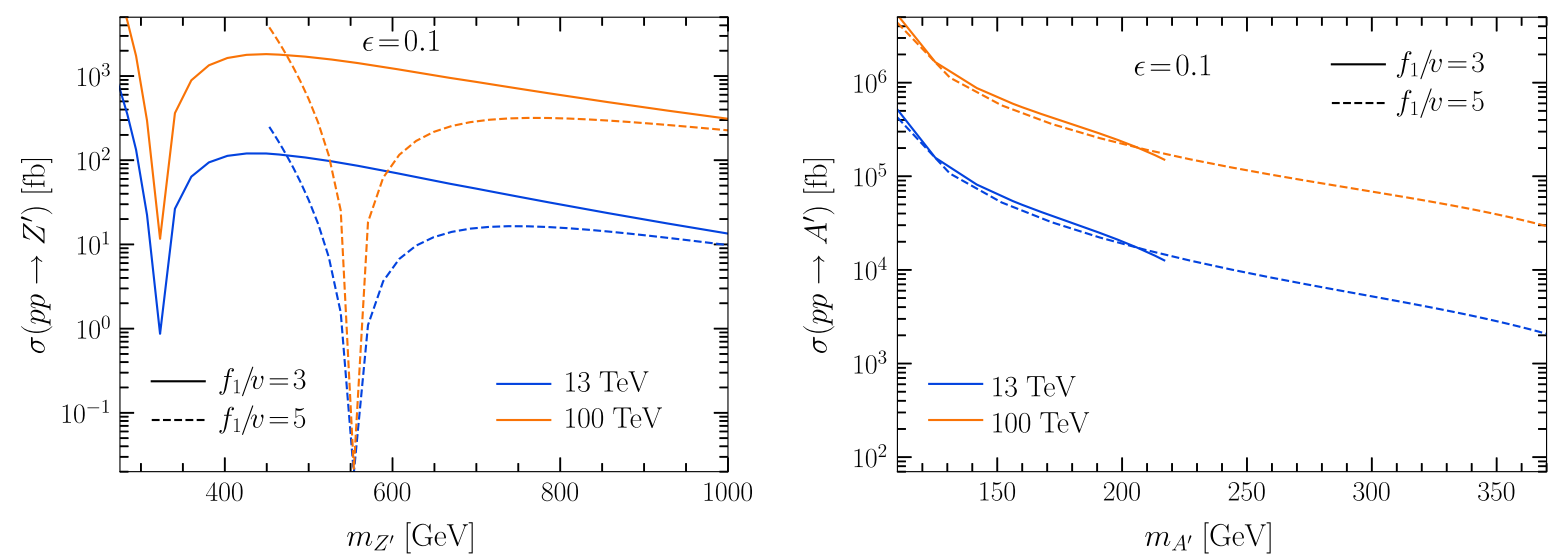

FIG. 5. Production cross section of the $Z^{\prime}$ (left) and $A^{\prime}$ (right) at the LHC and at a future $100 \mathrm{TeV}$ hadron collider in the T2HDM for $\epsilon=0.1$ and $f_{1} / v=3$ and 5 . For $m_{\bar{A}^{\prime}}=s_{2 W} m_{\bar{Z}^{\prime}}$ the alignment of $Z^{\prime}$ is orthogonal to twin hypercharge. This closes the portal to the SM sector, reducing production.

suppressing the production cross section and branching into visible states. At the same point, of course, the $A^{\prime}$ is perfectly aligned with twin hypercharge, so its coupling to the visible sector is enhanced.
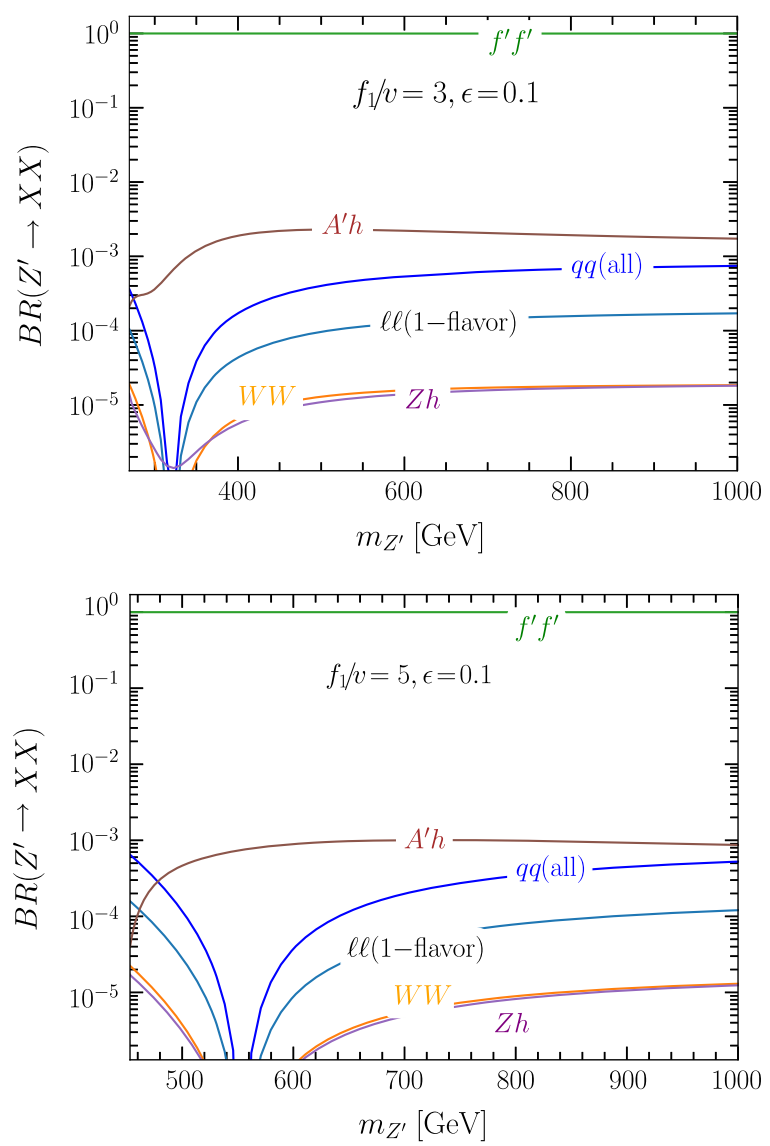

To analyze the experimental signals, we transform to a basis in which the mass and kinetic terms of the $A^{\prime}$ and $Z^{\prime}$, as well as those of the SM photon and $Z$, are diagonal. As in the THPM case, this is accomplished by first performing a
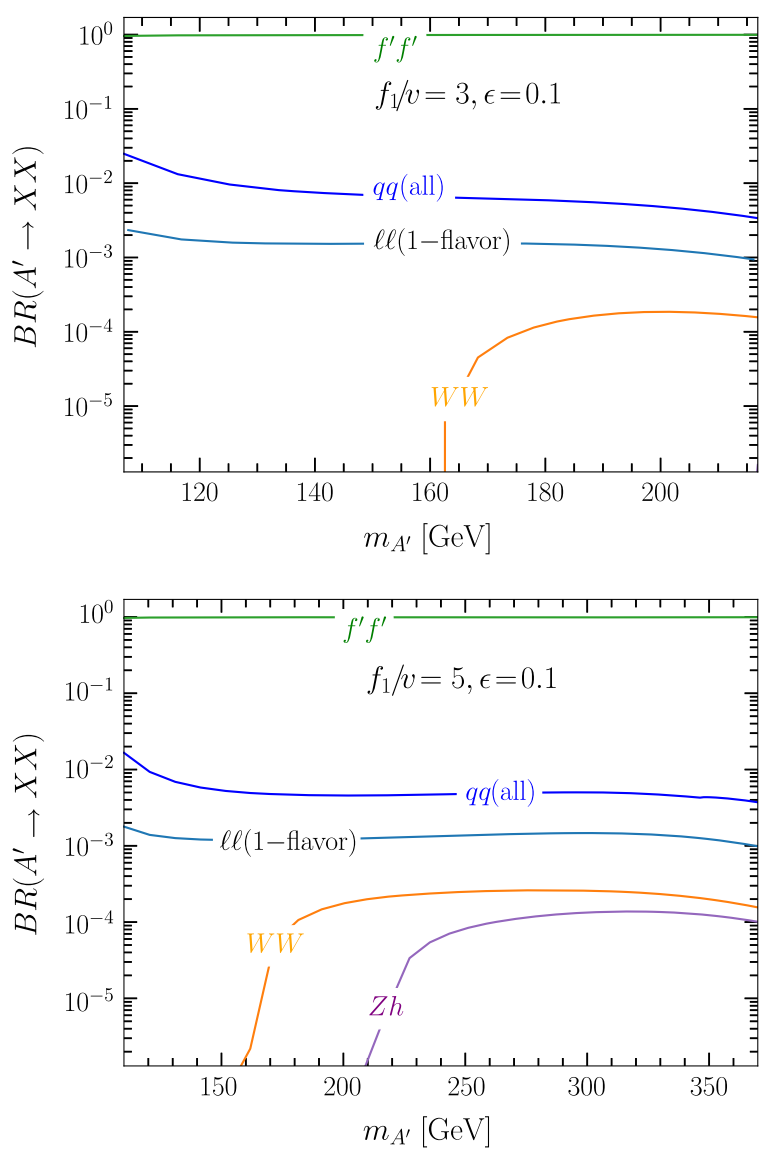

FIG. 6. Branching ratios of the $Z^{\prime}$ (left) and $A^{\prime}$ (right) in the T2HDM for $\epsilon=0.1$ and $f_{1} / v=3$ and 5 . The blue curve is for a single SM lepton flavor, while the dark blue curve corresponds to all six quark flavors. For $m_{\bar{A}^{\prime}}=s_{2 W} m_{\bar{Z}^{\prime}}$ the alignment of $Z^{\prime}$ is orthogonal to twin hypercharge. This closes the portal to the SM sector, reducing branching to visible states. 
shift of the SM hypercharge gauge boson $B$ and then rotating into the mass basis, as discussed in Appendix A. Once the kinetic and mass terms of the vector bosons have been diagonalized, it is straightforward to calculate their couplings to fermions. The details can be found in Appendix B. In Figs. 5 and 6 we plot the production cross sections and branching fractions, respectively, of the $A^{\prime}$ and $Z^{\prime}$ in the T2HDM. We see that the production cross section for the $A^{\prime}$ is always larger than for the $Z^{\prime}$. The branching fraction of the $A^{\prime}$ to SM final states is also greater than for the $Z^{\prime}$. These features, along with its lighter mass, enhance the prospects for $A^{\prime}$ discovery while inhibiting $Z^{\prime}$ discovery. We also see a striking feature when $m_{\bar{A}^{\prime}}=s_{2 W} m_{\bar{Z}^{\prime}}$, where the $Z^{\prime}$ is orthogonal to twin hypercharge and its couplings to $\mathrm{SM}$ states vanish.

\section{EXISTING CONSTRAINTS}

In this section we determine the constraints on the vector boson sector of the MTH model from direct searches and from precision electroweak observables. These are displayed in Fig. 7, the left plot showing the THPM model and the right plot the T2HDM. The top (bottom) row is for the benchmark of $f_{(1)} / v=3(5)$.

(i) LEP analyses place strong limits on any new physics contributions that affect the properties of the $Z$ boson. Quantities that can be determined directly from measurements at the $Z$ pole include the total width of the $Z$ boson, as well as the cross sections into various SM final states through the $Z$ resonance. Given these measurements, theory can be employed to fit other parameters in a straightforward way. In particular, theory relates the resonant dilepton production cross section and the total width to the leptonic width of the $Z$ in a model-independent way. This can therefore be used to constrain models of new physics. A different combination of the direct $Z$-pole measurements allows the invisible width of the $Z$ boson to be determined, again in a model-independent way, which provides a powerful independent constraint on new physics. We therefore begin our study of existing constraints on the MTH model with these two LEP Z-pole measurements.
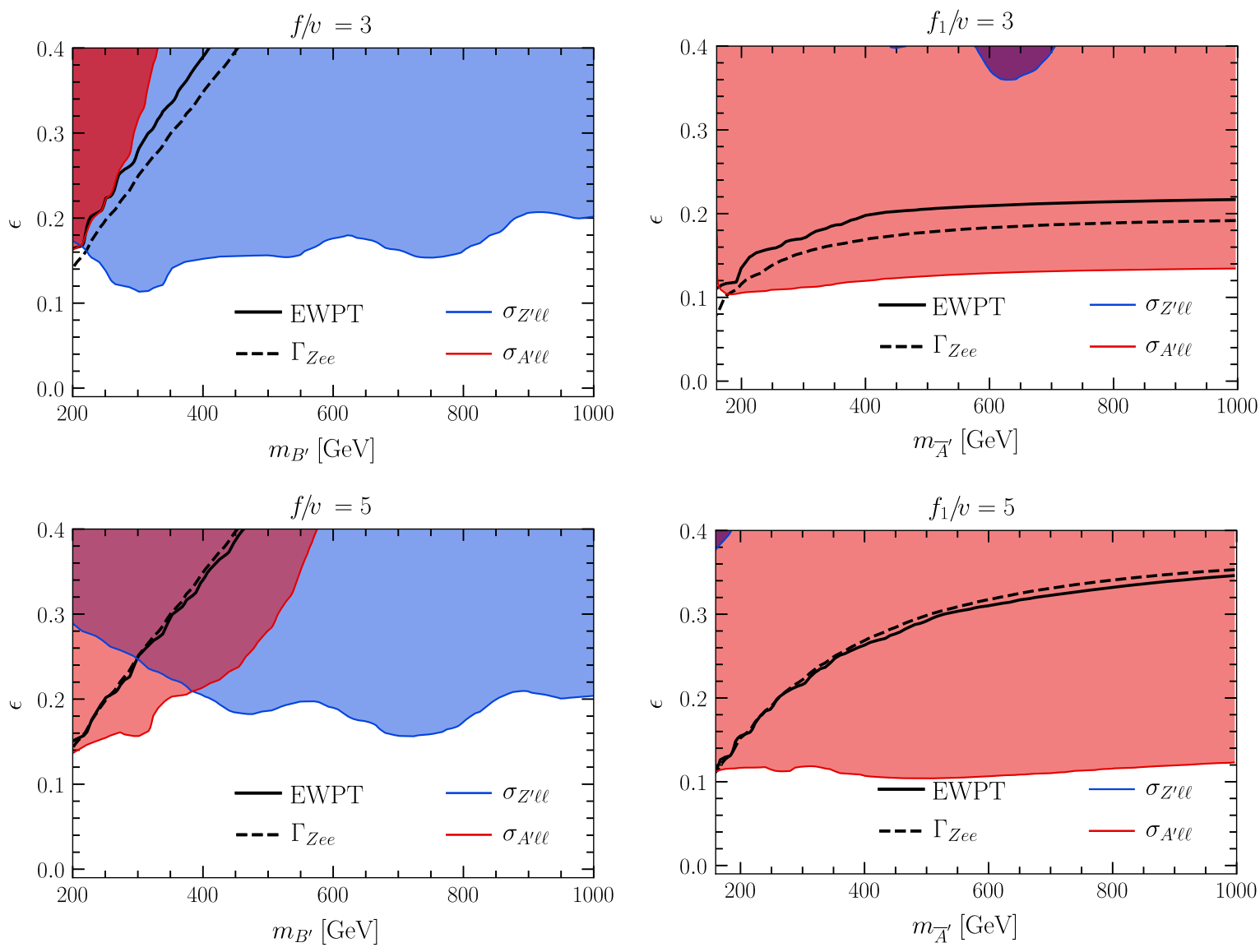

FIG. 7. Compilation of constraints on the MTH model in terms of $\epsilon$ and either $m_{B^{\prime}}$ (THPM model on the left) or $m_{\bar{A}^{\prime}}$ (T2HDM on the right) with $f_{(1)} / v=3(5)$ in the top (bottom) row. Indirect constraints are shown in black. The 13 TeV LHC dilepton resonance search bounds on the $A^{\prime}\left(Z^{\prime}\right)$ are shown in red (blue). 
The measured invisible width of the $Z$ boson is $\Gamma_{\text {Inv }}^{\mathrm{LEP}}=499.0 \pm 1.5 \mathrm{MeV}$ [79]. The predicted value for the SM contribution is $\Gamma_{\text {Inv }}^{\mathrm{SM}}=501.3 \pm 0.6 \mathrm{MeV}$ [80]. Therefore, the preferred central value and associated uncertainty of any potential new physics contribution are given by

$$
\Delta \Gamma_{\text {Inv }}=-2.2 \pm 1.6 \mathrm{MeV} .
$$

In our models, there are two contributions to $\Delta \Gamma_{\text {Inv }}$ : a reduction due to the change in the couplings to the neutrinos and a strictly positive contribution due to $Z$ decays to kinematically accessible twin states. We find that the reduction in the SM width dominates, so the MTH invisible $Z$ width is generically smaller than the SM prediction, bringing it closer to the LEP measurement. Consequently, this does not significantly constrain either model.

The modification of the $Z$-boson coupling to electrons in the MTH model, as well as to the twin sector states, also constrains the parameter space. The LEP bound on the partial width of $Z \rightarrow e^{+} e^{-}$is given by [79]

$$
\Gamma\left(Z \rightarrow e^{+} e^{-}\right)=83.91 \pm 0.12 \mathrm{MeV} .
$$

We require this partial width to be within the $2 \sigma$ range from $83.67 \mathrm{MeV}$ to $84.15 \mathrm{MeV}$ in our model, which excludes the regions above the black dashed lines in Fig. 7.

(ii) Electroweak precision data as encoded in the oblique parameters [81], in particular the $T$ parameter, also constrain the relevant parameter space. The mixing among the neutral vector bosons alters the relation between the $Z$ boson mass and the $W$ boson mass in the SM. The leading order correction is given in Eq. (A20). Using the notation of Ref. [82], we have

$$
\frac{\alpha T}{1+\alpha T}=1-\frac{m_{Z}^{2}}{m_{Z_{0}}^{2}} .
$$

Here $m_{Z_{0}}$ is the $Z$ boson mass in the SM, and $\alpha$ is the fine-structure constant evaluated at $m_{Z_{0}}$.

While this effect is the largest, there are other contributions to both the $S$ and $T$ parameters from the reduction of the Higgs coupling to SM states. These effects have been calculated in the general case [83]. Applied to our models, we obtain

$$
T \approx-\frac{3 v^{2}}{8 \pi c_{W}^{2} f_{(1)}^{2}} \ln \frac{\Lambda_{\mathrm{UV}}}{m_{Z_{0}}}, \quad S \approx \frac{v^{2}}{6 \pi f_{(1)}^{2}} \ln \frac{\Lambda_{\mathrm{UV}}}{m_{Z_{0}}} .
$$

While this contribution to $T$ is smaller than Eq. (3.3), it also has the opposite sign, reducing the deviation. We use the current bound on the $T$ parameter, leaving $U$ as a free parameter [84]. For $\Lambda_{\mathrm{UV}}=5 \mathrm{TeV}$ the contribution to $S$ in Eq. (3.4) varies from about 0.02 to 0.008 , while the contribution to $T$ varies from -0.06 to -0.02 , as $f / v$ changes from 3 to 5 . In this case, the 95\% exclusion contours require $T<0.14$ and 0.13 , respectively. The excluded region is shown as the area above the solid black line in Fig. 7.

We see from the figure that both the $Z \rightarrow e^{+} e^{-}$ partial width and the $T$-parameter bounds are extremely restrictive in the T2HDM case. This occurs because, in the T2HDM, the lighter state continues to have sizable couplings to the SM even as $m_{\bar{A}^{\prime}}$ gets large, so the bound remains almost unchanged even as the $Z^{\prime}$ becomes heavy. In contrast, in the THPM model, the lighter $A^{\prime}$ decouples from the SM sector in the limit that the mass of the $Z^{\prime}$ is large, so the bounds on this scenario fall off as $m_{Z^{\prime}}$ increases.

(iii) Direct searches at the LHC place strong bounds on dilepton resonances produced from a $\bar{q}-q$ initial state. We use the ATLAS limits $[85,86]$ and employ MADGRAPH5 [87] to simulate backgrounds. The $A^{\prime}$ and $Z^{\prime}$ production and decay at the LHC are simulated using the MSTW PDFs [88]. Our analysis is performed at the parton level since we are only dealing with leptons in the final state. In Fig. 7 we show the 95\% exclusion region from the resonant $Z^{\prime}$ (blue shaded) and $A^{\prime}$ (red shaded) production with subsequent decays to electrons and muons as a function of $m_{B^{\prime}}$ (left) or $m_{\bar{A}^{\prime}}$ (right) and $\epsilon$ and $f_{(1)} / v=3(5)$ on the top (bottom) row.

As we saw in Fig. 1, the $A^{\prime}$ mass in the THPM model asymptotes to $\left(v^{\prime} / v\right) m_{W}$ as $m_{B^{\prime}}$ gets large, with similar behavior in the T2HDM. However, in the THPM case both the production cross section (see Fig. 2) and dilepton branching ratio of the $A^{\prime}$ (see Fig. 3) fall off rapidly in this region, where $A^{\prime}$ decouples from the SM. Therefore, the $A^{\prime}$ is unconstrained for larger values of $m_{B^{\prime}}$. Conversely, in the T2HDM the production (see Fig. 5) and dilepton branching fraction of the $A^{\prime}$ (see Fig. 6) remain sizable as $m_{\bar{A}^{\prime}}$ increases, making the bound from $A^{\prime}$ searches strong over the entire mass range of interest. In the THPM model the $Z^{\prime}$ limits persist, dominating the limits at higher $m_{B^{\prime}}$. In the T2HDM, however, the $Z^{\prime}$ decouples from the $\mathrm{SM}$ at $m_{\bar{A}^{\prime}}=s_{2 W} m_{\bar{Z}^{\prime}}$, causing the bound to vanish at this point. Even away from this point, the couplings of the $Z^{\prime}$ to the SM are, in general, smaller than those of the $A^{\prime}$, so the bounds on the T2HDM are dominated by the limits on the $A^{\prime}$. 


\section{DISCOVERY PROSPECTS}

Now that the constraints on this framework have been mapped out, we determine the discovery and exclusion reach of the HL-LHC and a $100 \mathrm{TeV}$ hadron collider. We estimate the sensitivity of future dilepton searches by a simple scaling up of existing results. As an example, if a run-II dilepton resonance search at a given mass can exclude a signal cross section of $\sigma_{S, 13}$ at $95 \%$ confidence level, then a similar search at a $100 \mathrm{TeV}$ collider will be sensitive to a cross section $\sigma_{S, 100}$ at the same confidence level, given by

$$
\sigma_{S, 100}=\sigma_{S, 13} \sqrt{\frac{\sigma_{\mathrm{BG} 100 \mathrm{TeV}}}{\sigma_{\mathrm{BG} 13 \mathrm{TeV}}}} \sqrt{\frac{\mathcal{L}_{13}}{\mathcal{L}_{100}}},
$$

where the square roots contain the ratios of the background cross sections at the two colliders, as well as the ratio of the luminosities of the two searches. We calculate the background cross sections at 13 and $100 \mathrm{TeV}$ at parton level in MADGRAPH. Our results for $f_{(1)} / v=3$ and 5 are shown in Fig. 8 for the HL-LHC and Fig. 9 for the FCC-hh with a luminosity of $3000 \mathrm{fb}^{-1}$, the THPM model on the left and the T2HDM on the right. As in the collider constraints of the previous section, we see that in the THPM model the sensitivity to $A^{\prime}$ falls off as $m_{B^{\prime}}$ increases, while the sensitivity to $Z^{\prime}$ persists. Conversely, in the T2HDM the $A^{\prime}$ sensitivity falls off only slowly with $m_{\bar{A}^{\prime}}$, while the sensitivity to the $Z^{\prime}$ is much weaker across the entire parameter range.

The result is that in the THPM model, it is typically the $Z^{\prime}$ channel that can be used to improve the limits on $\epsilon$ at the LHC and FCC, gaining a factor of a few over the constraints shown in Fig. 7, whereas in the T2HDM the $A^{\prime}$ drives the sensitivity. This must be considered quite impressive, since for these resonances both the production cross section and the branching ratio into SM states scale roughly as $\epsilon^{2}$.

In the models we consider, the vector boson sector can be specified by three parameters: $f_{(1)} / v, \epsilon$, and either $m_{B^{\prime}}$ or $m_{\bar{A}^{\prime}}$. Therefore, measuring four or more independent observables can confirm the underlying structure. The observables in question can be the masses of $A^{\prime}$ and $Z^{\prime}$ and their resonant dilepton production rates, $\sigma_{\ell \ell . A^{\prime}}$ and $\sigma_{\ell \ell, Z^{\prime}}$. This is a goal that could be pursued at the LHC alone. Both vectors would appear as resonances in the
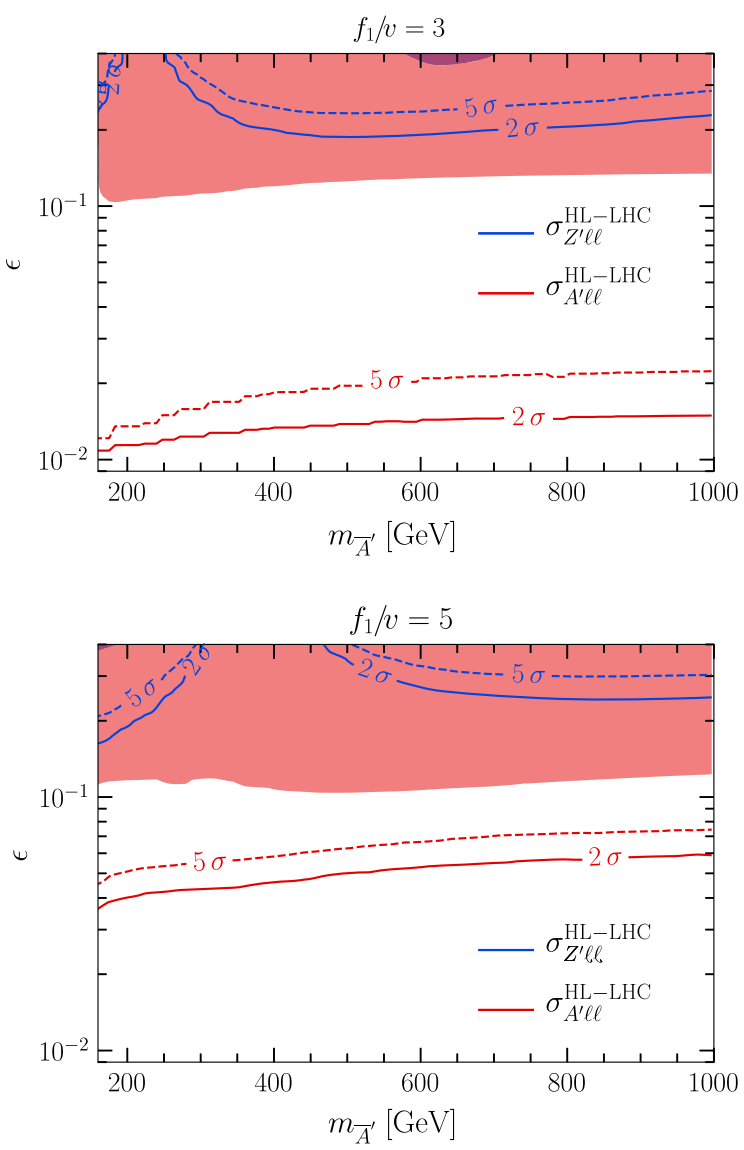

FIG. 8. Projected $\sigma$ contours of the $Z^{\prime}$ (blue) and $A^{\prime}$ (red) at the HL-LHC for a luminosity of $3000 \mathrm{fb}^{-1}$ for $f_{(1)} / v=3(5)$ on the top (bottom) row. The THPM model is on the left, and the T2HDM is on the right. The regions excluded by current LHC searches for the twin bosons are shaded in the corresponding color. 

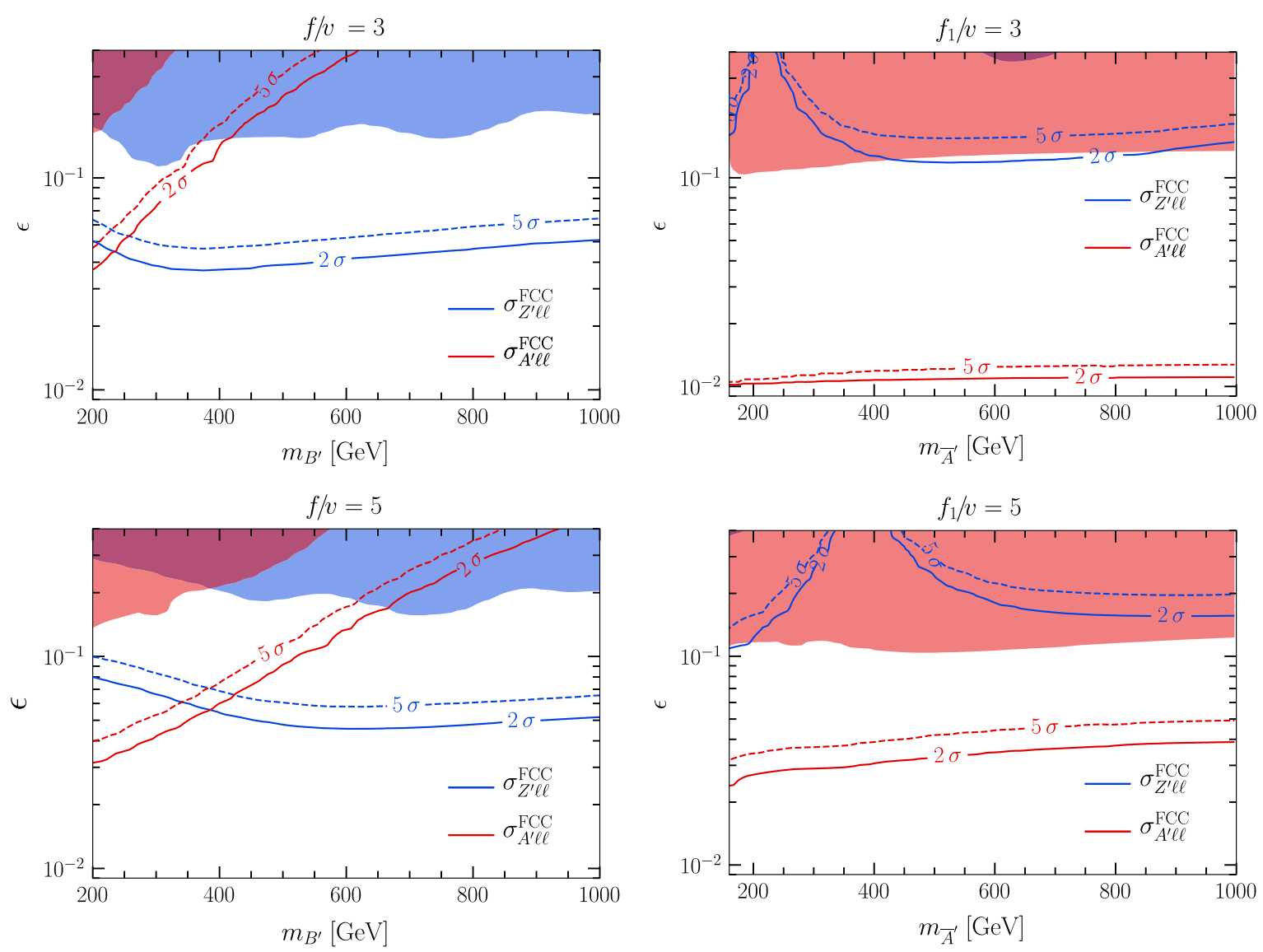

FIG. 9. Projected $\sigma$ contours of the $Z^{\prime}$ (blue) and $A^{\prime}$ (red) at a FCC hadron machine for a luminosity of $3000 \mathrm{fb}^{-1}$ for $f_{(1)} / v=3(5)$ on the top (bottom) row. The THPM model is on the left, and the T2HDM is on the right. The regions excluded by current LHC searches for the twin bosons are shaded in the corresponding color.

dilepton spectrum. Then, measuring both masses and any one event rate would completely specify the parameters of the model. A measurement of the other rate then provides a test of the theory.

From Fig. 8, we see that this is possible at the LHC for the THPM model. Although some part of the parameter space where both the $A^{\prime}$ and the $Z^{\prime}$ can be discovered is already ruled out by the current bounds on the $Z^{\prime}$, a sizable region remains. In the T2HDM, however, this is not the case. Although the LHC has excellent sensitivity to the $A^{\prime}$, the regions where the $Z^{\prime}$ can be discovered at the HL-LHC are already ruled out by current bounds on the $A^{\prime}$.

Before a FCC-hh machine begins taking data, it is very likely that the tunnel will be used for a lepton collider. The FCC-ee is projected to measure some Higgs couplings to better than $1 \%$, an order of magnitude beyond the HL-LHC [89]. In particular, the coupling between the Higgs and $Z$ bosons may be measured to $0.3 \%$ at $95 \%$ confidence [90]. We therefore expect that before the FCC-hh begins taking data, precise measurements of the Higgs couplings will already have determined $v / f_{(1)}$ to high accuracy. In both the THPM model and the T2HDM, the hadron machine can then completely specify the twin vector boson sector by measuring the mass and dilepton event rate of just one of the vectors, either the $A^{\prime}$ or the $Z^{\prime}$. In each of these models, the mass and dilepton event rate of the second vector are then predicted, and a targeted search may be made to test these theories. From Fig. 8 we see that in the THPM model, there are regions of parameter space where both the $A^{\prime}$ and $Z^{\prime}$ can be discovered at the FCC-hh. However, in the T2HDM the regions where the $Z^{\prime}$ can be discovered are already ruled out by the current LHC bounds on the $A^{\prime}$, so the model cannot be tested with this approach.

\section{CONCLUSIONS}

MTH models offer a simple solution to the little hierarchy problem without introducing new states charged under the SM gauge groups. The MTH framework predicts both a twin photon and a twin $Z$ boson. These states can interact with the SM through kinetic mixing between the hypercharge gauge boson of the SM and its mirror counterpart. If the twin photon is massive, this mixing can be sizable without violating the current experimental bounds. This portal can then be exploited by the LHC and future colliders to discover the twin vector bosons. 
We have determined the bounds on the $A^{\prime}$ and $Z^{\prime}$ vector boson masses in a model in which the twin hypercharge gauge boson has a Proca mass, and also in a twin version of the two Higgs doublet model. In most of the parameter space, LHC searches for neutral gauge bosons constrain the mixing parameter $\epsilon \lesssim 0.1$ for both the THPM and T2HDM. The HL-LHC and a $100 \mathrm{TeV}$ collider with $3000 \mathrm{fb}^{-1}$ of luminosity can improve on the current bounds by a factor of a few in most of parameter space. In the THPM, in some regions of parameter space the HL-LHC or a future $100 \mathrm{TeV}$ machine can discover both the neutral twin sector vector bosons. By measuring both the masses of these particles and the event rates into dilepton final states, these colliders can test the THPM model. However, in the case of the T2HDM, the current LHC bounds on the $A^{\prime}$ already exclude the parameter space in which the HLLHC or FCC-hh would be expected to discover the $Z^{\prime}$, and therefore we cannot test the model with this approach.

\section{ACKNOWLEDGMENTS}

We are grateful to Aqeel Ahmed and Anson Hook for helpful discussions. Z. C. is supported in part by the National Science Foundation under Grant No. PHY1620074. Z. C. would like to thank the Fermilab Theory Group for hospitality during the completion of this work. Z.C.'s stay at Fermilab was supported by the Fermilab Intensity Frontier program and by the Universities Research Association through their Visiting Scholar program. The research of C. K. is supported by the National Science Foundation Grant No. PHY-1620610. S. N. is supported by Vrije Universiteit Brussel through the Strategic Research Program "High Energy Physics" and also supported by FWO under the EOS-be.h Project No. 30820817. C. B. V. is supported by Department of Energy Grant No. DE-SC-0009999.

\section{APPENDIX A: MODEL DETAILS AND DIAGONALIZATION}

The SM and twin sectors couple through the hypercharge portal. Consequently, the SM photon and $Z$ boson mix with their twin counterparts, and the physical eigenstates are linear combinations of these states. In this Appendix we diagonalize the Lagrangian for these vector bosons and determine the physical eigenstates. The full process of diagonalization involves several steps, which we take in turn. First, we define twin sector states analogous to the familiar photon and $Z$ boson in the SM,

$\bar{Z}_{\mu}^{\prime} \equiv c_{W} W_{\mu}^{\prime 3}-s_{W} B_{\mu}^{\prime}, \quad \bar{A}_{\mu}^{\prime} \equiv s_{W} W_{\mu}^{\prime 3}+c_{W} B_{\mu}^{\prime}$,

with $\cos \theta_{W} \equiv c_{W}$ and $\sin \theta_{W} \equiv s_{W}$, where $\theta_{W}$ is the weak mixing angle. For each of the models treated in the text, the Proca mass model (THPM) and twin two Higgs doublet model (T2HDM), this leads to a mass mixing matrix,

$\frac{1}{2}\left(\begin{array}{ll}\bar{A}_{\mu}^{\prime} & \bar{Z}_{\mu}^{\prime}\end{array}\right)\left(\begin{array}{cc}c_{W}^{2} m_{B^{\prime}}^{2} & -s_{W} c_{W} m_{B^{\prime}}^{2} \\ -s_{W} c_{W} m_{B^{\prime}}^{2} & m_{\bar{Z}^{\prime}}^{2}+s_{W}^{2} m_{B^{\prime}}^{2}\end{array}\right)\left(\begin{array}{c}\bar{A}_{\mu}^{\prime} \\ \bar{Z}_{\mu}^{\prime}\end{array}\right)$ THPM,

$\frac{1}{2}\left(\begin{array}{ll}\bar{A}_{\mu}^{\prime} & \bar{Z}_{\mu}^{\prime}\end{array}\right)\left(\begin{array}{cc}m_{\bar{A}^{\prime}}^{2} & m_{\bar{A}^{\prime}}^{2} / t_{2 W} \\ m_{\bar{A}^{\prime}}^{2} / t_{2 W} & m_{\bar{Z}^{\prime}}^{2}+m_{\bar{A}^{\prime}}^{2} / t_{2 W}^{2}\end{array}\right)\left(\begin{array}{c}\bar{A}_{\mu}^{\prime} \\ \bar{Z}_{\mu}^{\prime}\end{array}\right)$ T2HDM.

We move to the diagonal basis, denoted with the subscript 0 , through the rotation matrix

$$
\left(\begin{array}{c}
Z_{0 \mu}^{\prime} \\
A_{0 \mu}^{\prime}
\end{array}\right)=\left(\begin{array}{cc}
\cos \phi & -\sin \phi \\
\sin \phi & \cos \phi
\end{array}\right)\left(\begin{array}{c}
\bar{A}_{\mu}^{\prime} \\
\bar{Z}_{\mu}^{\prime}
\end{array}\right) .
$$

The eigenvalues are given by

$$
\begin{aligned}
m_{Z_{0}^{\prime}, A_{0}^{\prime}}^{2}= & \frac{m_{\bar{Z}^{\prime}}^{2}+m_{B^{\prime}}^{2}}{2} \\
& \pm \frac{1}{2} \sqrt{\left(m_{B^{\prime}}^{2}-m_{\bar{Z}^{\prime}}^{2}\right)^{2}+4 s_{W}^{2} m_{B^{\prime}}^{2} m_{\bar{Z}^{\prime}}^{2}} \text { THPM, }
\end{aligned}
$$

$$
\begin{aligned}
m_{Z_{0}^{\prime}, A_{0}^{\prime}}^{2}= & \frac{m_{\bar{Z}^{\prime}}^{2} s_{2 W}^{2}+m_{\bar{A}^{\prime}}^{2}}{2 s_{2 W}^{2}} \\
& \pm \frac{1}{2} \sqrt{\left(m_{\bar{Z}^{\prime}}^{2}+c_{4 W} \frac{m_{\bar{A}^{\prime}}^{2}}{s_{2 W}^{2}}\right)^{2}+4 \frac{m_{\bar{A}^{\prime}}^{4}}{t_{2 W}^{2}}} \text { T2HDM, }
\end{aligned}
$$

and the mixing angle is

$$
\sin 2 \phi=\frac{m_{B^{\prime}}^{2} s_{2 W}}{\sqrt{\left(m_{B^{\prime}}^{2}-m_{\bar{Z}^{\prime}}^{2}\right)^{2}+4 s_{W}^{2} m_{B^{\prime}}^{2} m_{\bar{Z}^{\prime}}^{2}}},
$$

for the THPM model. The T2HDM has

$$
\sin 2 \phi=-\frac{m_{\bar{A}^{\prime}}^{2} s_{4 W}}{\sqrt{\left(m_{\bar{Z}^{\prime}}^{2} s_{2 W}^{2}+c_{4 W} m_{\bar{A}^{\prime}}^{2}\right)^{2}+s_{4 W}^{2} m_{\bar{A}^{\prime}}^{4}}} .
$$

In the discussion that follows, the results are expressed in terms of the angle $\phi$. Therefore, all the results we obtain apply to both models, with the relation to the model parameters determined by the equations above.

The Lagrangian for the neutral vector bosons in the visible and twin sectors takes the form 


$$
\begin{aligned}
\mathcal{L} \supset & -\frac{1}{4} W_{0 \mu \nu} W_{0}^{\mu \nu}-\frac{1}{4} A_{0 \mu \nu}^{\prime} A_{0}^{\prime \mu \nu}-\frac{1}{4} Z_{0 \mu \nu}^{\prime} Z_{0}^{\prime \mu \nu}-\frac{1}{4} B_{0 \mu \nu} B_{0}^{\mu \nu} \\
& +\frac{m_{Z_{0}}^{2}}{2} Z_{0 \mu} Z_{0}^{\mu}+\frac{\epsilon c_{T}}{2} B_{0 \mu \nu} Z^{\prime 0 \mu \nu}-\frac{\epsilon s_{T}}{2} B_{0 \mu \nu} A^{\prime 0 \mu \nu} \\
& +\frac{m_{A_{0}^{\prime}}^{2}}{2} A_{0 \mu}^{\prime} A_{0}^{\prime \mu}+\frac{m_{Z_{0}^{\prime}}^{2}}{2} Z_{0 \mu}^{\prime} Z_{0}^{\prime \mu},
\end{aligned}
$$

where we have used the definitions

$$
c_{T} \equiv \cos \left(\theta_{W}-\phi\right), \quad s_{T} \equiv \sin \left(\theta_{W}-\phi\right) .
$$

Here

$$
X_{\mu \nu} \equiv \partial_{\mu} X_{\nu}-\partial_{\nu} X_{\mu}
$$

for each Abelian vector $X$, with the non-Abelian generalization used when appropriate. Note that for the THPM model, the limit $m_{B^{\prime}} \gg m_{\bar{Z}^{\prime}}$ leads to $\phi \approx \theta_{W}$. Then, in this limit $c_{T} \approx 1$ and $s_{T} \approx 0$. Therefore, we expect $A^{\prime}$ to decouple from the SM for large $m_{B^{\prime}}$. In the T2HDM, the limit $m_{\bar{A}^{\prime}}=s_{2 W} m_{\bar{Z}^{\prime}}$ leads to $\phi=\theta_{W}-\pi / 2$. In this limit $c_{T}=0$ and $s_{T}=-1$, meaning that $Z^{\prime}$ decouples from the SM for this particular ratio of the twin sector VEVs.

Returning to the Lagrangian in Eq. (A9), we note that we may completely unmix all the kinetic terms by the following transformation:

$$
\left(\begin{array}{c}
B_{0 \mu} \\
Z_{0 \mu}^{\prime} \\
A_{0 \mu}^{\prime}
\end{array}\right)=\left(\begin{array}{ccc}
1 & c_{T} \epsilon(1+\alpha) & -\epsilon s_{T}(1+\alpha) \\
0 & 1+c_{T}^{2} \alpha & -c_{T} s_{T} \alpha \\
0 & -c_{T} s_{T} \alpha & 1+s_{T}^{2} \alpha
\end{array}\right)\left(\begin{array}{c}
B_{1 \mu} \\
Z_{1 \mu}^{\prime} \\
A_{1 \mu}^{\prime}
\end{array}\right),
$$

where

$$
\alpha=-1+\frac{1}{\sqrt{1-\epsilon^{2}}} .
$$

In the absence of kinetic mixing the eigenstates in the visible sector are given by

$$
Z_{0 \mu} \equiv c_{W} W_{\mu}^{3}-s_{W} B_{0 \mu}, \quad A_{0 \mu} \equiv s_{W} W_{\mu}^{3}+c_{W} B_{0 \mu} .
$$

We define

$$
Z_{1 \mu} \equiv c_{W} W_{\mu}^{3}-s_{W} B_{1 \mu}, \quad A_{1 \mu} \equiv s_{W} W_{\mu}^{3}+c_{W} B_{1 \mu} .
$$

We can express $Z_{0 \mu}$ and $A_{0 \mu}$ in terms of $Z_{1 \mu}, A_{1 \mu}, Z_{1 \mu}^{\prime}$ and $A_{1 \mu}^{\prime}$,

$$
\begin{aligned}
& Z_{0 \mu}=Z_{1 \mu}-\epsilon s_{W}(1+\alpha)\left(c_{T} Z_{1 \mu}^{\prime}-s_{T} A_{1 \mu}^{\prime}\right), \\
& A_{0 \mu}=A_{1 \mu}+\epsilon c_{W}(1+\alpha)\left(c_{T} Z_{1 \mu}^{\prime}-s_{T} A_{1 \mu}^{\prime}\right) .
\end{aligned}
$$

Expressed in terms of $Z_{1 \mu}, A_{1 \mu}, Z_{1 \mu}^{\prime}$ and $A_{1 \mu}^{\prime}$, the Lagrangian is given by

$$
\begin{aligned}
\mathcal{L} \supset & -\frac{1}{4} Z_{1 \mu \nu} Z_{1}^{\mu \nu}-\frac{1}{4} A_{1 \mu \nu} A_{1}^{\mu \nu}-\frac{1}{4} A_{1 \mu \nu}^{\prime} A_{1}^{\prime \mu \nu}-\frac{1}{4} Z_{1 \mu \nu}^{\prime} Z_{1}^{\prime \mu \nu} \\
& +\frac{m_{Z_{0}^{\prime}}^{2}}{2}\left[\left(1+c_{T}^{2} \alpha\right) Z_{1 \mu}^{\prime}-c_{T} s_{T} \alpha A_{1 \mu}^{\prime}\right]^{2} \\
& +\frac{m_{A_{0}^{\prime}}^{2}}{2}\left[\left(1+s_{T}^{2} \alpha\right) A_{1 \mu}^{\prime}-c_{T} s_{T} \alpha Z_{1 \mu}^{\prime}\right]^{2} \\
& +\frac{m_{Z_{0}}^{2}}{2}\left[Z_{1 \mu}-s_{W} \epsilon(1+\alpha)\left(c_{T} Z_{1 \mu}^{\prime}-s_{T} A_{1 \mu}^{\prime}\right)\right]^{2} .
\end{aligned}
$$

This leads to a mass matrix with the characteristic equation

$$
\begin{aligned}
& \left(\lambda-m_{Z_{0}}^{2}\right)\left(\lambda-m_{Z_{0}^{\prime}}^{2}\right)\left(\lambda-m_{A_{0}^{\prime}}^{2}\right) \\
& \quad+\epsilon^{2} \lambda\left(\lambda-c_{W}^{2} m_{Z_{0}}^{2}\right)\left(\lambda-s_{T}^{2} m_{Z_{0}^{\prime}}^{2}-c_{T}^{2} m_{A_{0}^{\prime}}^{2}\right)=0 .
\end{aligned}
$$

Finding the exact mass eigenvalues from this equation is not simple, but we note a few features. First, in the limit $\epsilon \rightarrow 0$ the eigenvalues are $m_{A_{0}^{\prime}}^{2}, m_{Z_{0}^{\prime}}^{2}$, and $m_{Z_{0}}^{2}$, as expected. Direct inspection of the eigenvalue equation also shows that the leading correction to these values arises at order $\epsilon^{2}$. These corrections are given by

$$
\begin{array}{r}
m_{Z}^{2}=m_{Z_{0}}^{2}+s_{W}^{2} \epsilon^{2} m_{Z_{0}}^{2}\left(\frac{c_{T}^{2} m_{Z_{0}}^{2}}{m_{Z_{0}}^{2}-m_{Z_{0}^{\prime}}^{2}}+\frac{s_{T}^{2} m_{Z_{0}}^{2}}{m_{Z_{0}}^{2}-m_{A_{0}^{\prime}}^{2}}\right), \\
m_{Z^{\prime}}^{2}=m_{Z_{0}^{\prime}}^{2}+\epsilon^{2} c_{T}^{2} m_{Z_{0}^{\prime}}^{2} \frac{m_{Z_{0}^{\prime}}^{2}-m_{Z_{0}}^{2} c_{W}^{2}}{m_{Z_{0}^{\prime}}^{2}-m_{Z_{0}}^{2}},
\end{array}
$$

$$
m_{A^{\prime}}^{2}=m_{A_{0}^{\prime}}^{2}+\epsilon^{2} s_{T}^{2} m_{A_{0}^{\prime}}^{2} \frac{m_{A_{0}^{\prime}}^{2}-m_{Z_{0}}^{2} c_{W}^{2}}{m_{A_{0}^{\prime}}^{2}-m_{Z_{0}}^{2}} .
$$

These formulas break down near mass degeneracies. At these points the mass matrix can be diagonalized numerically. One can also work out the corresponding eigenvectors and hence the similarity transform to the diagonal basis at order $\epsilon^{2}$.

However, we can see the leading effects of the mixing by just working to linear order in $\epsilon$. We obtain

$$
\begin{array}{r}
Z_{0 \mu}=Z_{\mu}\left[1-\frac{\epsilon^{2} m_{Z_{0}}^{4} s_{W}^{2}}{2}\left(\frac{s_{T}^{2}}{\left(m_{A_{0}^{\prime}}^{2}-m_{Z}^{2}\right)^{2}}+\frac{c_{T}^{2}}{\left(m_{Z_{0}^{\prime}}^{2}-m_{Z_{0}}^{2}\right)^{2}}\right)\right] \\
-\epsilon s_{W}\left[Z_{\mu}^{\prime} \frac{c_{T} m_{Z_{0}^{\prime}}^{2}}{m_{Z_{0}^{\prime}}^{2}-m_{Z_{0}}^{2}}-A_{\mu}^{\prime} \frac{s_{T} m_{A_{0}^{\prime}}^{2}}{m_{A_{0}^{\prime}}^{2}-m_{Z_{0}}^{2}}\right], \quad(\mathrm{A} 23) \\
A_{0 \mu}=A_{\mu}+\epsilon c_{W}\left(c_{T} Z_{\mu}^{\prime}-s_{T} A_{\mu}^{\prime}\right),
\end{array}
$$




$$
\begin{aligned}
& Z_{0 \mu}^{\prime}=Z_{\mu}^{\prime}+Z_{\mu} \frac{\epsilon s_{W} c_{T} m_{Z_{0}}^{2}}{m_{Z_{0}^{\prime}}^{2}-m_{Z_{0}}^{2}}, \\
& A_{0 \mu}^{\prime}=A_{\mu}^{\prime}-Z_{\mu} \frac{\epsilon s_{W} s_{T} m_{Z_{0}}^{2}}{m_{A_{0}^{\prime}}^{2}-m_{Z_{0}}^{2}} .
\end{aligned}
$$

We have kept effects of order $\epsilon^{2}$ in the expression for the $Z$ because the correction to the $Z$ couplings to this order is important to obtain the constraints in Sec. III. The results in the body of the paper are based on a numerical analysis, so the formulas in this Appendix are intended only to provide a qualitative understanding.

\section{APPENDIX B: VECTOR TO FERMION COUPLINGS}

In this section we record the couplings of the gauge bosons to the fermions of the two sectors and determine their partial widths. The visible photon's couplings are simple,

$$
g_{A f \bar{f}}=g s_{W} Q=e Q, \quad g_{A f^{\prime} \bar{f}^{\prime}}=0 .
$$

The couplings of the $Z, A^{\prime}$ and $Z^{\prime}$ bosons to fermions and twin fermions can be parametrized as

$$
\begin{aligned}
\mathcal{L}_{\text {Int }}= & g_{Z f \bar{f}} \bar{f} \gamma^{\mu} f Z_{\mu}+g_{A^{\prime} f \bar{f}} \bar{f} \gamma^{\mu} f A^{\prime}+g_{Z^{\prime} f \bar{f}} \bar{f} \gamma^{\mu} f Z^{\prime} \\
& +g_{Z f^{\prime} \bar{f}^{\prime}} \bar{f}^{\prime} \gamma^{\mu} f^{\prime} Z_{\mu}+g_{A^{\prime} f^{\prime} \bar{f}^{\prime}} \bar{f}^{\prime} \gamma^{\mu} f^{\prime} A^{\prime}+g_{Z^{\prime} f^{\prime} \bar{f}^{\prime}} \bar{f}^{\prime} \gamma^{\mu} f^{\prime} Z^{\prime}
\end{aligned}
$$

The couplings of the massive neutral gauge bosons to the SM fermions are given by

$$
\begin{gathered}
g_{Z f \bar{f}}=\frac{g}{c_{W}}\left\{\left(T^{3}-Q s_{W}^{2}\right)\left[1-\frac{\epsilon^{2} m_{Z_{0}}^{4} s_{W}^{2}}{2}\left(\frac{s_{T}^{2}}{\left(m_{A_{0}^{\prime}}^{2}-m_{Z}^{2}\right)^{2}}+\frac{c_{T}^{2}}{\left(m_{Z_{0}^{\prime}}^{2}-m_{Z_{0}}^{2}\right)^{2}}\right)\right]+Y \epsilon^{2} m_{Z_{0}}^{2} s_{W}^{2}\left(\frac{s_{T}^{2}}{m_{A_{0}^{\prime}}^{2}-m_{Z}^{2}}+\frac{c_{T}^{2}}{m_{Z_{0}^{\prime}}^{2}-m_{Z}^{2}}\right)\right\}, \\
g_{Z^{\prime} f \bar{f}}=-\frac{g}{c_{W}} \epsilon s_{W} c_{T}\left[\left(T^{3}-Q s_{W}^{2}\right) \frac{m_{Z_{0}}^{2}}{m_{Z_{0}^{\prime}}^{2}-m_{Z_{0}}^{2}}-Y\right], \\
g_{A^{\prime} f \bar{f}}=\frac{g}{c_{W}} \epsilon s_{W} s_{T}\left[\left(T^{3}-Q s_{W}^{2}\right) \frac{m_{Z_{0}}^{2}}{m_{A_{0}^{\prime}}^{2}-m_{Z_{0}}^{2}}-Y\right] .
\end{gathered}
$$

The couplings of the massive neutral gauge bosons to the fermions of the twin sector are

$$
\begin{gathered}
g_{Z f^{\prime} \bar{f}^{\prime}}=\frac{-g}{c_{W}} \epsilon m_{Z_{0}}^{2} s_{W}\left\{\left[\cos \phi\left(T^{3}-Q s_{W}^{2}\right)+Q s_{W} c_{W} \sin \phi\right] \frac{s_{T}}{m_{A_{0}^{\prime}}^{2}-m_{Z_{0}}^{2}}+\left[\sin \phi\left(T^{3}-Q s_{W}^{2}\right)-Q s_{W} c_{W} \cos \phi\right] \frac{c_{T}}{\left.m_{Z_{0}^{\prime}}^{2}-m_{Z_{0}}^{2}\right\},}\right. \\
g_{Z^{\prime} f^{\prime} \bar{f}^{\prime}}=\frac{-g}{c_{W}}\left[\sin \phi\left(T^{3}-Q s_{W}^{2}\right)-Q s_{W} c_{W} \cos \phi\right], \\
g_{A^{\prime} f^{\prime} f^{\prime}}=\frac{g}{c_{W}}\left[\cos \phi\left(T^{3}-Q s_{W}^{2}\right)+Q s_{W} c_{W} \sin \phi\right] .
\end{gathered}
$$

In calculating the branching fractions of the various gauge bosons, the following formulas are of use. The decay width of the vector boson $V\left(Z, A^{\prime}, Z^{\prime}\right)$ to fermions is given by

$$
\Gamma(V \rightarrow f f)=\frac{N_{c} m_{V}}{24 \pi} \sqrt{1-4 \frac{m_{f}^{2}}{m_{V}^{2}}}\left[\left(g_{L}^{f 2}+g_{R}^{f 2}\right)\left(1-\frac{m_{f}^{2}}{m_{V}^{2}}\right)+6 g_{L}^{f} g_{R}^{f} \frac{m_{f}^{2}}{m_{V}^{2}}\right] .
$$

The neutral vector bosons also have a decay width to $Z h$, where $h$ denotes the Higgs. This contribution to the decay width arises from the $h Z_{0} Z_{0}$ and $h \bar{Z}^{\prime} \bar{Z}^{\prime}$ terms in the Lagrangian. This leads to the terms $g_{A^{\prime} Z h} A_{\mu}^{\prime} Z^{\mu} h$ and $g_{Z^{\prime} Z h} Z_{\mu}^{\prime} Z^{\mu} h$ with

$$
g_{A^{\prime} Z h}=2 \epsilon s_{W} \frac{m_{Z_{0}}^{2}}{v_{\mathrm{EW}}}\left(1-\frac{v_{\mathrm{EW}}^{2}}{2 f_{(1)}^{2}}\right)\left[s_{T} \frac{m_{A_{0}^{\prime}}^{2}+m_{Z_{0}}^{2} \cos ^{2} \phi}{m_{A_{0}^{\prime}}^{2}-m_{Z_{0}}^{2}}+c_{T} \frac{m_{Z_{0}}^{2} \cos \phi \sin \phi}{m_{Z_{0}^{\prime}}^{2}-m_{Z_{0}}^{2}}\right] \text {, }
$$




$$
\begin{aligned}
g_{Z^{\prime} Z h}= & -2 \epsilon s_{W} \frac{m_{Z_{0}}^{2}}{v_{\mathrm{EW}}}\left(1-\frac{v_{\mathrm{EW}}^{2}}{2 f_{(1)}^{2}}\right)\left[c_{T} \frac{m_{Z_{0}^{\prime}}^{2}+m_{Z_{0}}^{2} \sin ^{2} \phi}{m_{Z_{0}^{\prime}}^{2}-m_{Z_{0}}^{2}}\right. \\
& \left.+s_{T} \frac{m_{Z_{0}}^{2} \cos \phi \sin \phi}{m_{A_{0}^{\prime}}^{2}-m_{Z_{0}}^{2}}\right] .
\end{aligned}
$$

The decay widths are then given by

$$
\begin{aligned}
\Gamma(V \rightarrow Z h) & =\frac{g_{V h Z}^{2}}{192 \pi} \frac{m_{V}}{m_{Z}^{2}} \lambda\left(1, x_{Z}, x_{h}\right)\left[\lambda^{2}\left(1, x_{Z}, x_{h}\right)+12 x_{Z}\right], \\
\lambda(a, b, c) & =\sqrt{a^{2}+b^{2}+c^{2}-2 a b-2 b c-2 a c}, \\
x_{Z} & =\frac{m_{Z}^{2}}{m_{V}^{2}}, \quad x_{h}=\frac{m_{h}^{2}}{m_{V}^{2}} .
\end{aligned}
$$

The decay of a vector $V$ into a pair of visible $W$ bosons arises from the usual $Z_{0} W W$ and $A_{0} W W$ vertices in the visible sector and by using Eqs. (A23) and (A24). The width is then given by

$$
\begin{aligned}
\Gamma(V \rightarrow W W) & =f(k) g_{V^{\prime} W W}^{2} \frac{m_{V}}{192 \pi}, \\
f(k) & =(1-4 k)^{\frac{3}{2}}\left(1+20 k+12 k^{2}\right) k^{-2}, \\
k & =\frac{m_{W}^{2}}{m_{V}^{2}} .
\end{aligned}
$$

Decays of the $Z^{\prime}$ into $W^{\prime} W^{\prime}$ have the same form, with the coupling arising from the $\bar{Z}^{\prime} W^{\prime} W^{\prime}$ and $\bar{A}^{\prime} W^{\prime} W^{\prime}$ vertices.
[1] G. Aad et al. (ATLAS Collaboration), Observation of a new particle in the search for the Standard Model Higgs boson with the ATLAS detector at the LHC, Phys. Lett. B 716, 1 (2012).

[2] S. Chatrchyan et al. (CMS Collaboration), Observation of a new boson at a mass of $125 \mathrm{GeV}$ with the CMS experiment at the LHC, Phys. Lett. B 716, 30 (2012).

[3] Z. Chacko, H.-S. Goh, and R. Harnik, The Twin Higgs: Natural Electroweak Breaking from Mirror Symmetry, Phys. Rev. Lett. 96, 231802 (2006).

[4] R. Barbieri, T. Gregoire, and L. J. Hall, Mirror world at the large hadron collider, arXiv:hep-ph/0509242.

[5] Z. Chacko, Y. Nomura, M. Papucci, and G. Perez, Natural little hierarchy from a partially goldstone twin Higgs, J. High Energy Phys. 01 (2006) 126.

[6] G. Burdman, Z. Chacko, H.-S. Goh, and R. Harnik, Folded supersymmetry and the LEP paradox, J. High Energy Phys. 02 (2007) 009.

[7] D. Poland and J. Thaler, The dark top, J. High Energy Phys. 11 (2008) 083.

[8] H. Cai, H.-C. Cheng, and J. Terning, A quirky little Higgs model, J. High Energy Phys. 05 (2009) 045.

[9] N. Craig, S. Knapen, and P. Longhi, Neutral Naturalness from Orbifold Higgs Models, Phys. Rev. Lett. 114, 061803 (2015).

[10] N. Craig, S. Knapen, and P. Longhi, The orbifold Higgs, J. High Energy Phys. 03 (2015) 106.

[11] B. Batell and M. McCullough, Neutrino masses from neutral top partners, Phys. Rev. D 92, 073018 (2015).

[12] C. Csáki, T. Ma, and J. Shu, Trigonometric Parity for Composite Higgs Models, Phys. Rev. Lett. 121, 231801 (2018).

[13] J. Serra and R. Torre, Neutral naturalness from the brotherHiggs model, Phys. Rev. D 97, 035017 (2018).

[14] T. Cohen, N. Craig, G. F. Giudice, and M. Mccullough, The hyperbolic Higgs, J. High Energy Phys. 05 (2018) 091.
[15] H.-C. Cheng, L. Li, E. Salvioni, and C. B. Verhaaren, Singlet scalar top partners from accidental supersymmetry, J. High Energy Phys. 05 (2018) 057.

[16] B. M. Dillon, Neutral-naturalness from a holographic $S O(6) / S O(5)$ composite Higgs model, Phys. Rev. D 99, 115008 (2019).

[17] L.-X. Xu, J.-H. Yu, and S.-H. Zhu, Minimal neutral naturalness model, arXiv:1810.01882.

[18] G. Burdman, Z. Chacko, R. Harnik, L. de Lima, and C. B. Verhaaren, Colorless top partners, a $125 \mathrm{GeV}$ Higgs, and the limits on naturalness, Phys. Rev. D 91, 055007 (2015).

[19] H. Beauchesne, K. Earl, and T. Grëgoire, The spontaneous $\mathbb{Z}_{2}$ breaking twin Higgs, J. High Energy Phys. 01 (2016) 130.

[20] J.-H. Yu, Radiative- $\mathbb{Z}_{2}$-breaking twin Higgs model, Phys. Rev. D 94, 111704 (2016).

[21] J.-H. Yu, A tale of twin Higgs: Natural twin two Higgs doublet models, J. High Energy Phys. 12 (2016) 143.

[22] T. H. Jung, Spontaneous twin symmetry breaking, arXiv: 1902.10978.

[23] B. Batell and C.B. Verhaaren, Breaking mirror twin hypercharge, arXiv:1904.10468.

[24] A. Falkowski, S. Pokorski, and M. Schmaltz, Twin SUSY, Phys. Rev. D 74, 035003 (2006).

[25] S. Chang, L. J. Hall, and N. Weiner, A supersymmetric twin Higgs, Phys. Rev. D 75, 035009 (2007).

[26] N. Craig and K. Howe, Doubling down on naturalness with a supersymmetric twin Higgs, J. High Energy Phys. 03 (2014) 140.

[27] A. Katz, A. Mariotti, S. Pokorski, D. Redigolo, and R. Ziegler, SUSY meets her twin, J. High Energy Phys. 01 (2017) 142.

[28] M. Badziak and K. Harigaya, Supersymmetric D-term twin Higgs, J. High Energy Phys. 06 (2017) 065.

[29] M. Badziak and K. Harigaya, Minimal non-Abelian supersymmetric twin Higgs, J. High Energy Phys. 10 (2017) 109. 
[30] M. Badziak and K. Harigaya, Asymptotically Free Natural Supersymmetric Twin Higgs Model, Phys. Rev. Lett. 120, 211803 (2018).

[31] Z. Berezhiani, Through the looking-glass: Alice's adventures in mirror world, From Fields to Strings: Circumnavigating Theoretical Physics (World Scientific, Singapore, 2005), pp. 2147-2195.

[32] M. Geller and O. Telem, Holographic Twin Higgs Model, Phys. Rev. Lett. 114, 191801 (2015).

[33] R. Barbieri, D. Greco, R. Rattazzi, and A. Wulzer, The composite twin Higgs scenario, J. High Energy Phys. 08 (2015) 161.

[34] M. Low, A. Tesi, and L.-T. Wang, Twin Higgs mechanism and a composite Higgs boson, Phys. Rev. D 91, 095012 (2015).

[35] P. Asadi, N. Craig, and Y.-Y. Li, Twin Turtles, J. High Energy Phys. 02 (2019) 138.

[36] R. Contino, D. Greco, R. Mahbubani, R. Rattazzi, and R. Torre, Precision tests and fine tuning in twin Higgs models, Phys. Rev. D 96, 095036 (2017).

[37] C. Csaki, M. Geller, O. Telem, and A. Weiler, The flavor of the composite twin Higgs, J. High Energy Phys. 09 (2016) 146.

[38] H.-C. Cheng, S. Jung, E. Salvioni, and Y. Tsai, Exotic quarks in twin Higgs models, J. High Energy Phys. 03 (2016) 074.

[39] H.-C. Cheng, E. Salvioni, and Y. Tsai, Exotic electroweak signals in the twin Higgs model, Phys. Rev. D 95, 115035 (2017).

[40] Z. G. Berezhiani and R. N. Mohapatra, Reconciling present neutrino puzzles: Sterile neutrinos as mirror neutrinos, Phys. Rev. D 52, 6607 (1995).

[41] Z. G. Berezhiani, A. D. Dolgov, and R. N. Mohapatra, Asymmetric inflationary reheating and the nature of mirror universe, Phys. Lett. B 375, 26 (1996).

[42] Z. Chacko, N. Craig, P. J. Fox, and R. Harnik, Cosmology in mirror twin Higgs and neutrino masses, J. High Energy Phys. 07 (2017) 023.

[43] N. Craig, S. Koren, and T. Trott, Cosmological signals of a mirror twin Higgs, J. High Energy Phys. 05 (2017) 038.

[44] M. Farina, Asymmetric twin dark matter, J. Cosmol. Astropart. Phys. 11 (2015) 017.

[45] R. Barbieri, L. J. Hall, and K. Harigaya, Minimal mirror twin Higgs, J. High Energy Phys. 11 (2016) 172.

[46] C. Csaki, E. Kuflik, and S. Lombardo, Viable twin cosmology from neutrino mixing, Phys. Rev. D 96, 055013 (2017).

[47] R. Barbieri, L. J. Hall, and K. Harigaya, Effective theory of flavor for minimal mirror twin Higgs, J. High Energy Phys. 10 (2017) 015.

[48] H.-C. Cheng, L. Li, and R. Zheng, Coscattering/coannihilation dark matter in a fraternal twin Higgs model, J. High Energy Phys. 09 (2018) 098.

[49] M. Farina, A. Monteux, and C. S. Shin, Twin mechanism for baryon and dark matter asymmetries, Phys. Rev. D 94, 035017 (2016).

[50] K. Earl, C. S. Fong, T. Gregoire, and A. Tonero, Mirror Dirac leptogenesis, arXiv:1903.12192.

[51] K. Fujikura, K. Kamada, Y. Nakai, and M. Yamaguchi, Phase transitions in twin Higgs models, J. High Energy Phys. 12 (2018) 018.
[52] Z. Chacko, D. Curtin, M. Geller, and Y. Tsai, Cosmological signatures of a mirror twin Higgs, J. High Energy Phys. 09 (2018) 163.

[53] N. Craig, S. Knapen, P. Longhi, and M. Strassler, The vector-like twin Higgs, J. High Energy Phys. 07 (2016) 002.

[54] N. Craig, A. Katz, M. Strassler, and R. Sundrum, Naturalness in the dark at the LHC, J. High Energy Phys. 07 (2015) 105.

[55] D. Curtin and C.B. Verhaaren, Discovering uncolored naturalness in exotic Higgs decays, J. High Energy Phys. 12 (2015) 072.

[56] C. Csaki, E. Kuflik, S. Lombardo, and O. Slone, Searching for displaced Higgs boson decays, Phys. Rev. D 92, 073008 (2015).

[57] N. Craig and A. Katz, The fraternal WIMP miracle, J. Cosmol. Astropart. Phys. 10 (2015) 054.

[58] I. Garcia Garcia, R. Lasenby, and J. March-Russell, Twin Higgs WIMP dark matter, Phys. Rev. D 92, 055034 (2015).

[59] I. Garcia Garcia, R. Lasenby, and J. March-Russell, Twin Higgs Asymmetric Dark Matter, Phys. Rev. Lett. 115, 121801 (2015).

[60] Y. Hochberg, E. Kuflik, and H. Murayama, Twin Higgs model with strongly interacting massive particle dark matter, Phys. Rev. D 99, 015005 (2019).

[61] J. Terning, C. B. Verhaaren, and K. Zora, Composite twin dark matter, Phys. Rev. D 99, 095020 (2019).

[62] A. Ahmed, Heavy Higgs of the twin Higgs models, J. High Energy Phys. 02 (2018) 048.

[63] Z. Chacko, C. Kilic, S. Najjari, and C. B. Verhaaren, Testing the scalar sector of the twin Higgs model at colliders, Phys. Rev. D 97, 055031 (2018).

[64] D. Buttazzo, D. Redigolo, F. Sala, and A. Tesi, Fusing vectors into scalars at high energy lepton colliders, J. High Energy Phys. 11 (2018) 144.

[65] C. Kilic, S. Najjari, and C. B. Verhaaren, Discovering the twin Higgs boson with displaced decays, Phys. Rev. D 99, 075029 (2019).

[66] S. Alipour-Fard, N. Craig, S. Gori, S. Koren, and D. Redigolo, The second Higgs at the lifetime frontier, arXiv: 1812.09315 .

[67] F. Bishara and C. B. Verhaaren, Singleton portals to the twin sector, J. High Energy Phys. 05 (2019) 016.

[68] S. Davidson, S. Hannestad, and G. Raffelt, Updated bounds on millicharged particles, J. High Energy Phys. 05 (2000) 003.

[69] H. Vogel and J. Redondo, Dark radiation constraints on minicharged particles in models with a hidden photon, J. Cosmol. Astropart. Phys. 02 (2014) 029.

[70] B. Holdom, Two U(1)'s and epsilon charge shifts, Phys. Lett. 166B, 196 (1986).

[71] K. S. Babu, C. F. Kolda, and J. March-Russell, Implications of generalized Z-Z-prime mixing, Phys. Rev. D 57, 6788 (1998).

[72] N. Arkani-Hamed and N. Weiner, LHC signals for a superunified theory of dark matter, J. High Energy Phys. 12 (2008) 104.

[73] S. A. Abel, M. D. Goodsell, J. Jaeckel, V. V. Khoze, and A. Ringwald, Kinetic mixing of the photon with hidden U(1)s in string phenomenology, J. High Energy Phys. 07 (2008) 124. 
[74] M. Goodsell, J. Jaeckel, J. Redondo, and A. Ringwald, Naturally light hidden photons in LARGE volume string compactifications, J. High Energy Phys. 11 (2009) 027.

[75] M. Goodsell, S. Ramos-Sanchez, and A. Ringwald, Kinetic mixing of U(1)s in heterotic orbifolds, J. High Energy Phys. 01 (2012) 021.

[76] R. Essig et al., Working Group Report: New light weakly coupled particles, in Proceedings, 2013 Community Summer Study on the Future of U.S. Particle Physics: Snowmass on the Mississippi (CSS2013): Minneapolis, MN, USA (2013).

[77] I. Hoenig, G. Samach, and D. Tucker-Smith, Searching for dilepton resonances below the $\mathrm{Z}$ mass at the LHC, Phys. Rev. D 90, 075016 (2014).

[78] D. Curtin, R. Essig, S. Gori, and J. Shelton, Illuminating dark photons with high-energy colliders, J. High Energy Phys. 02 (2015) 157.

[79] C. Patrignani et al. (Particle Data Group), Review of particle physics, Chin. Phys. C 40, 100001 (2016).

[80] M. Carena, A. de Gouvea, A. Freitas, and M. Schmitt, Invisible Z boson decays at e+ e- colliders, Phys. Rev. D 68, 113007 (2003).

[81] M.E. Peskin and T. Takeuchi, A New Constraint on a Strongly Interacting Higgs Sector, Phys. Rev. Lett. 65, 964 (1990).

[82] T. Appelquist, B. A. Dobrescu, and A. R. Hopper, Nonexotic neutral gauge bosons, Phys. Rev. D 68, 035012 (2003).

[83] A. Falkowski, F. Riva, and A. Urbano, Higgs at last, J. High Energy Phys. 11 (2013) 111.
[84] M. Baak, J. Cuth, J. Haller, A. Hoecker, R. Kogler, K. Mnig, M. Schott, J. Stelzer, and (Gfitter Group), The global electroweak fit at NNLO and prospects for the LHC and ILC, Eur. Phys. J. C 74, 3046 (2014).

[85] M. Aaboud et al. (ATLAS Collaboration), Search for new high-mass phenomena in the dilepton final state using $36 \mathrm{fb}^{-1}$ of proton-proton collision data at $\sqrt{s}=13 \mathrm{TeV}$ with the ATLAS detector, J. High Energy Phys. 10 (2017) 182.

[86] G. Aad et al. (ATLAS Collaboration), Search for high-mass dilepton resonances using $139 \mathrm{fb}^{-1}$ of $p p$ collision data collected at $\sqrt{s}=13 \mathrm{TeV}$ with the ATLAS detector, Phys. Lett. B 796, 68 (2019).

[87] J. Alwall, R. Frederix, S. Frixione, V. Hirschi, F. Maltoni, O. Mattelaer, H. S. Shao, T. Stelzer, P. Torrielli, and M. Zaro, The automated computation of tree-level and next-toleading order differential cross sections, and their matching to parton shower simulations, J. High Energy Phys. 07 (2014) 79 .

[88] A. D. Martin, W. J. Stirling, R. S. Thorne, and G. Watt, Parton distributions for the LHC, Eur. Phys. J. C 63, 189 (2009).

[89] S. Dawson et al., Working Group Report: Higgs Boson, in Proceedings of the 2013 Community Summer Study on the Future of U.S. Particle Physics: Snowmass on the Mississippi (CSS2013): Minneapolis, MN, USA (2013).

[90] M. Bicer et al. (TLEP Design Study Working Group), First look at the physics case of TLEP, J. High Energy Phys. 01 (2014) 164. 